\title{
Spontaneous Spin Bifurcations and Ferromagnetic Phase Transitions in a Spinor Exciton-Polariton Condensate
}

\author{
H. Ohadi, ${ }^{1, *}$ A. Dreismann, ${ }^{1}$ Y. G. Rubo, ${ }^{2}$ F. Pinsker, ${ }^{3,4}$ Y. del Valle-Inclan Redondo, ${ }^{1}$ \\ S. I. Tsintzos, ${ }^{5}$ Z. Hatzopoulos, ${ }^{5,6}$ P. G. Savvidis, ${ }^{1,5,7}$ and J. J. Baumberg ${ }^{1, \dagger}$ \\ ${ }^{1}$ Department of Physics, Cavendish Laboratory, University of Cambridge, \\ Cambridge CB3 OHE, United Kingdom \\ ${ }^{2}$ Instituto de Energías Renovables, Universidad Nacional Autónoma de México, \\ Temixco, Morelos 62580, Mexico \\ ${ }^{3}$ Department of Applied Mathematics and Theoretical Physics, \\ University of Cambridge, Cambridge CB3 OWA, United Kingdom \\ ${ }^{4}$ Clarendon Laboratory, University of Oxford, Parks Road, Oxford OX1 3PU, United Kingdom \\ ${ }^{5}$ Foundation for Research and Technology-Hellas, Institute of Electronic Structure and Laser, \\ 71110 Heraklion, Crete, Greece \\ ${ }^{6}$ Department of Physics, University of Crete, 71003 Heraklion, Crete, Greece \\ ${ }^{7}$ Department of Materials Science and Technology, University of Crete, 71003 Heraklion, Crete, Greece \\ (Received 12 March 2015; revised manuscript received 29 April 2015; published 8 July 2015)

\begin{abstract}
We observe a spontaneous parity breaking bifurcation to a ferromagnetic state in a spatially trapped excitonpolariton condensate. At a critical bifurcation density under nonresonant excitation, the whole condensate spontaneously magnetizes and randomly adopts one of two elliptically polarized (up to $95 \%$ circularly polarized) states with opposite handedness of polarization. The magnetized condensate remains stable for many seconds at $5 \mathrm{~K}$, but at higher temperatures, it can flip from one magnetic orientation to another. We optically address these states and demonstrate the inversion of the magnetic state by resonantly injecting 100fold weaker pulses of opposite spin. Theoretically, these phenomena can be well described as spontaneous symmetry breaking of the spin degree of freedom induced by different loss rates of the linear polarizations.
\end{abstract}

DOI: 10.1103/PhysRevX.5.031002

\section{INTRODUCTION}

Condensation of exciton-polaritons (polaritons) spontaneously breaks the global phase symmetry [1-5]. Owing to their easy optical interrogation, high-speed (ps) interactions, and macroscopic coherence (over hundreds of microns) [6], polariton condensates are excellent candidates to probe and exploit for sensing $[7,8]$, spinoptronics [9-11], new optoelectronic devices [12-14], and quantum simulators [15]. The driven-dissipative multicomponent polariton system can undergo additional bifurcations and condense into states that are not eigenstates of the singleparticle Hamiltonian but many-body states with reduced symmetry [16,17]. Thus, we should expect that twocomponent exciton-polariton condensates can also show spontaneous symmetry-breaking bifurcations in their

\footnotetext{
ho278@cam.ac.uk

†jb12@cam.ac.uk
}

Published by the American Physical Society under the terms of the Creative Commons Attribution 3.0 License. Further distribution of this work must maintain attribution to the author(s) and the published article's title, journal citation, and DOI.
Subject Areas: Condensed Matter Physics, Magnetism,

Nonlinear Dynamics,

Semiconductor Physics

polarization state. Spin studies of microcavity polaritons have been of great interest in recent years [18-29]. However, spontaneous symmetry-breaking bifurcation of spin has not been observed before.

Here, we demonstrate spontaneous magnetization in an exciton-polariton condensate, as a direct result of bifurcations in the spin degree of freedom. Utilizing an optically trapped geometry, condensates spontaneously emerge in either of two discrete spin-polarized states that are stable for many seconds, $>10^{10}$ longer than their formation time. These states emit highly circularly polarized coherent light (up to 95\%) and have opposite circular polarizations. The condensate stochastically condenses in a left- or rightcircularly polarized state, with an occurrence likelihood that can be controlled by the ellipticity of the nonresonant pump. The two spin-polarized states can be initialized and switched from one state to another with weak resonant optical pulses. Our system has potential applications in sensing, optical spin memories, and spin switches, and it can be implemented for studying long-range spin interactions in polariton condensate lattices.

This article is structured as follows: In Sec. I, we review trapped polariton condensates and the current 
understanding of polarization in untrapped polariton condensates. In Sec. II, we present the key theme of this work, which is the spontaneous buildup of stochastic circular polarization. In Sec. III, we propose a theoretical framework for the phenomena discussed in this work. We show that stochastic circular polarization is a signature of spontaneous parity breaking. In Sec. IV, we time resolve the coherent driving of the spin, with resonant excitation. We furthermore investigate the stability of the spin-polarized states against thermal noise and conclude in Sec. V.

Exciton-polaritons are spinor particles formed by the strong coupling of excitons in a semiconductor quantum well with photons in the microcavity in which they are embedded [30]. We create optically trapped polariton condensates by nonresonant excitation of a semiconductor microcavity membrane [see Fig. 1(a) and Appendix A]. The excitation beam is shaped into a four-spot pattern [shown by dashed circles in Fig. 1(b)]. The shortwavelength, continuous wave $(\mathrm{CW})$, linearly polarized pump injects an electron-hole plasma at each pump spot, which rapidly relaxes to form excitons, in the process, losing all phase information. These reservoir excitons then scatter into polariton states via multiple phonon-polariton and stimulated polariton-polariton collisions [31], and they feed the zero-momentum ground state at the center of the trap. Because of their large effective mass, excitons typically diffuse only very small distances and stay within $1 \mu \mathrm{m}$ of the pump spots. Microcavity polaritons, however, are 10000 times lighter, giving longer diffusion lengths. Driven by their repulsive excitonic interactions, polaritons can thus travel large distances away from the pump spots

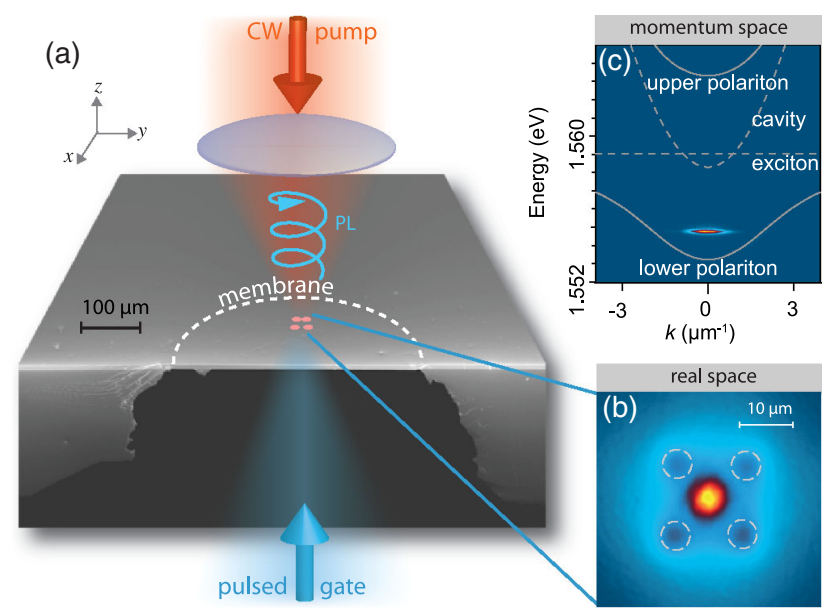

FIG. 1. (a) Scanning electron microscopy image of a membrane microcavity, cleaved across the middle. The membrane allows resonant excitation from the back side of the cavity. (b) Above threshold, hot polaritons condense into a single coherent state located at the center of the trap (pump spots shown by dashed circles), which is found at $k_{\|}=0$. (c) The spin state of the condensate is determined by studying the emission polarization. within their lifetime [32]. Once the density inside the trap exceeds the condensation threshold, a macroscopically coherent condensate is formed [Fig. 1(c)]. Because the condensate overlaps only weakly with the pump spots, it shows a narrower linewidth and less decoherence than unconfined condensates [33]. The optical trapping method used here is similar to optical lattices in cold atomic systems [34] but with the major difference that the optical potential also provides gain $[32,35,36]$.

Polaritons in quantum-well microcavities have two $J_{z}= \pm 1$ (spin-up or spin-down) projections of their total angular momentum along the growth axis of the structure, which correspond to right- and left-circularly polarized photons emitted by the cavity, respectively. When the excitation is linearly polarized, an equal population of spin-up and spin-down excitons forms in the reservoir. An initially spin-balanced reservoir, in the absence of pinning to any crystallographic axis, is expected to give a condensate with a stochastic linear polarization [37]. In most experiments with polariton lasers, the condensates have been found to be linearly polarized along one of the crystallographic axes $[1,2]$. Nevertheless, in some cases, a circularly polarized polariton lasing has also been observed $[5,19,23,38,39]$. Formation of a circularly polarized condensate is usually associated with the effects of TE-TM splitting, or bosonic amplification of the seed polarization of condensates. In all these cases, a circularly polarized condensate is observed when the symmetry between the spin-up and spin-down polaritons has been explicitly broken in some fashion, by either the pumping geometry (seeding with a circularly polarized pump) or by the imposed rotation of the Stokes parameters due to polarization splitting. As a result, the observed circular polarization was never stochastic (i.e., it is fixed each time the condensate is excited, instead of being different on each realization).

\section{SPONTANEOUS BUILDUP OF CIRCULAR POLARIZATION}

Our experiments reveal a completely different behavior to previous reports on the polarization of polariton condensates. We observe a strong degree of circular polarization $60 \%<\left|s_{z}\right|<95 \%$ [Fig. 2(a)] when the excitation is linearly polarized (to better than 1 in $10^{5}$ ), which is stable for many seconds [Fig. 2(b)]. The condensate stochastically adopts either of two opposite elliptical-polarization states in each realization of the experiment. We call these two states the spin-up $\left(s_{\uparrow}\right)$ and spin-down $\left(s_{\downarrow}\right)$ states. By mapping the polarization of the photoluminescence $(\mathrm{PL})$, we measure the polarization vector $s_{x, y, z}=$ $\left(I_{H, D, \circlearrowright}-I_{V, A, \circlearrowleft}\right) /\left(I_{H, D, \circlearrowright}+I_{V, A, \circlearrowleft}\right)$, where $I$ is the measured intensity for horizontal $(H)$, vertical $(V)$, diagonal $(D)$, antidiagonal $(A)$, right-circular ( $)$, and left-circular (S) polarizations. We measure all components of the 


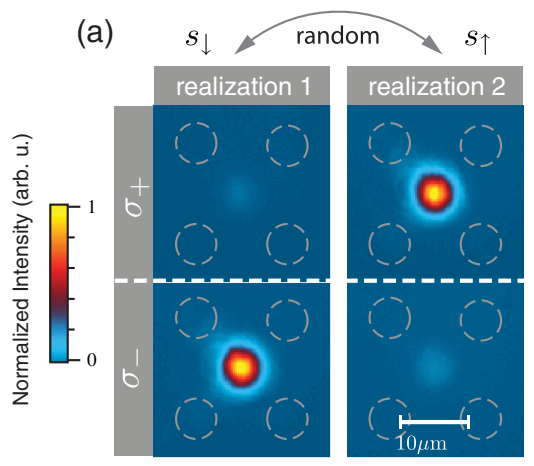

(b)

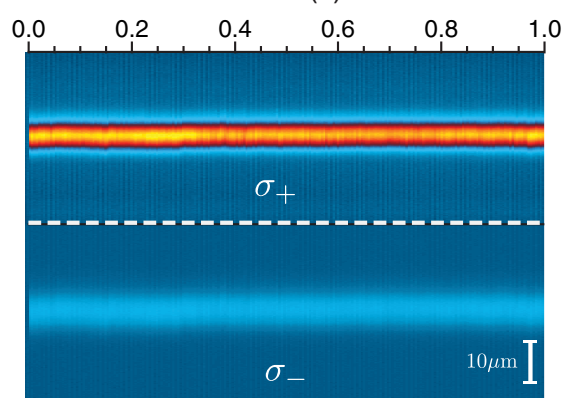

(c)

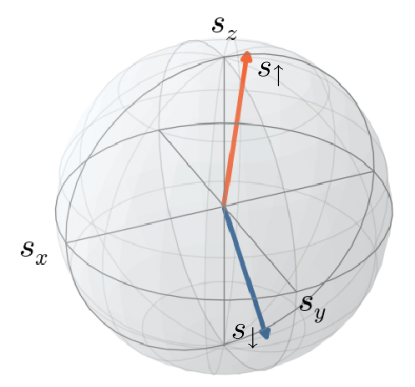

FIG. 2. (a) Polarization-resolved spatial image of the only two realizations observed in a single trapped condensate in a four-spot trapping geometry. (b) At $T=5 \mathrm{~K}$, the spin-polarized states remain stable for many seconds. (c) Simultaneously measured components of the pseudospin for 1000 realizations. The total degree of polarization is $0.93 \pm 0.03$, and the two average pseudospin states are $s_{\downarrow}=[-0.22,0.19,-0.94]$ (blue vector) and $s_{\uparrow}=[-0.22,-0.14,0.96]$ (orange vector). The measurement error for each component is $<5 \%$, and the variance is about $1 \%$.

polarization vector (pseudospin) simultaneously and plot the mean of the spin-up and spin-down states on the Poincaré sphere separately for 1000 realizations [Fig. 2(c)]. In each realization, the wave function of the condensate spontaneously collapses into one of the two discrete spin-polarized states which have opposing circular and diagonal components, marked by blue and orange vectors in Fig. 2(c). The linear axis along which the pseudospin flips (marked here as diagonal) does not depend on the geometry of the trap, and it changes direction with the position of the condensate on the sample.

To demonstrate that the buildup of circular polarization is truly spontaneous, we illuminate the sample with a longduration pulse of $9 \mathrm{~ms}$ using an acousto-optic modulator (AOM). A condensate builds up and picks a random state (e.g., $s_{\downarrow}$ ) and stays in that particular state as long as the pump pulse lasts [Fig. 3(a)]. We then repeat the same measurement, but this time, we modulate the pump intensity so that a condensate is created and destroyed every $2 \mu \mathrm{s}$. Figure $3(\mathrm{~b})$ shows how the condensate, although stable for many seconds, picks a random polarization at every successive realization due to random initial conditions at the onset of condensation. In this case, the parity symmetry is broken spontaneously. The buildup of circular polarization is independent of the precise position on the sample, of the sample orientation, of the pump spot orientation, and of the polariton detuning.

We can also explicitly break the symmetry in our experiments by changing the ellipticity of the pump laser and measure the contrast of the occurrence frequency of spin-up $\left(f_{\uparrow}\right)$ to spin-down $\left(f_{\downarrow}\right)$ condensate realizations, $\xi=\left(f_{\uparrow}-f_{\downarrow}\right) /\left(f_{\uparrow}+f_{\downarrow}\right)$. The probability of a realization resulting in state $f_{\uparrow \downarrow}$ is equal to $(1 \pm \xi) / 2$. Figure 3(c) shows $\xi$ as a function of the pump circular polarization $\left(s_{z, \text { pump }}\right)$ averaged over 1000 realizations for each point. As the ellipticity of the pump is increased from linear $\left(s_{z, \text { pump }}=0\right)$ to right circular $\left(s_{z, \text { pump }}>0\right)$, the probability of creating a condensate in the spin-up state
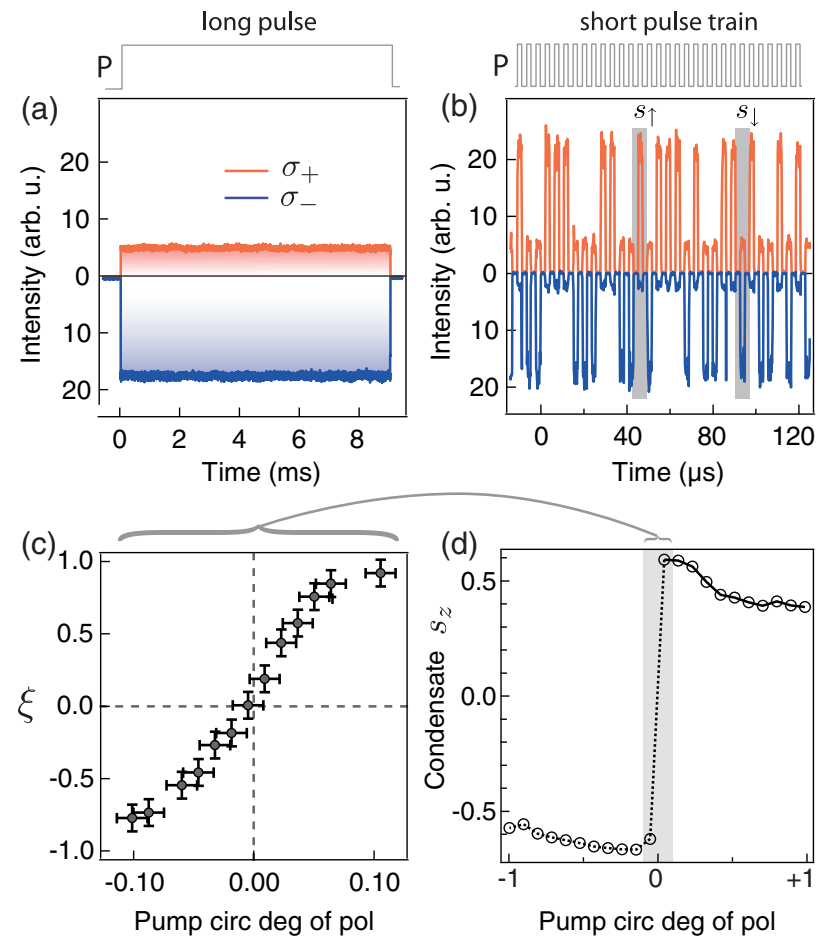

FIG. 3. (a) Emitted circular $\sigma_{+}$and $\sigma_{-}$intensities for a condensate realization when $P>P_{c}$, created by a long pulse (marked by $P$ ). The condensate randomly picks the $s_{\downarrow}$ state here. Note the opposite axis directions for $\sigma_{+}$and $\sigma_{-}$components. (b) Same as (a), with pump intensity modulated by a square wave (shown on top by $P$ ). A cut from the middle of a 9-ms exposure is shown, where each square pulse corresponds to a single realization. The condensate randomly picks $s_{\uparrow}$ or $s_{\downarrow}$ states (marked by grey rectangles). (c) Occurrence contrast $\xi$ of spin-up and spindown realizations measured in (b) vs the ellipticity of the pump. (d) Measured circular polarization of condensate vs pump ellipticity. Dashed lines mark the region where the condensate initializes stochastically in spin-up or spin-down states in each realization. 
increases; conversely, for the left-circularly polarized pump $\left(s_{z, \text { pump }}<0\right)$, the probability of creating a spin-down condensate increases. With a linearly polarized pump, we have an equal probability of creating a spin-up or spindown condensate. Although the pump laser is nonresonant with the final polariton states, the initially created carrier spin is not entirely randomized during their multiple carrier-carrier and exciton-phonon scatterings [5,28,39,40]. As a result of this incomplete spin relaxation of the excited carriers, changing the ellipticity of the pump breaks the symmetry of the condensate toward the same circular polarization as that of the pump. For pump circular polarizations far greater than 0.1 , the condensate is formed deterministically in the same polarization state as that of the pump [Fig. 3(d)].

An interesting question here is why we observe the spontaneous buildup of circular polarization, as opposed to the linear polarization that is widely reported in the literature $[1,2]$. The key difference between the experiments presented in this work and other studies of polariton condensation lies in the excitation geometry. For our trapped condensates, the pump and condensate are spatially separated, which critically reduces the contaminating interactions between the condensate and reservoir. The large interaction between untrapped condensates and the unpolarized exciton reservoir results in spin-flip scattering of polaritons with reservoir excitons. If there is a depolarized reservoir on top of the condensate, the spin-flip scattering processes minimize any imbalance between circular components of the condensate. This minimization leads to quenching of the buildup of circular polarization, forcing the polaritons to condense only with linear polarization. Moreover, it has been shown previously that, because of a smaller overlap with the reservoir, trapped condensates have a smaller linewidth than untrapped condensates [33]. Our careful studies with temperature and our theoretical calculations (see Sec. IV D) show how spin noise in the system results in spin flipping of the condensate. The spin-flip rate scales exponentially with noise. Larger linewidth untrapped condensates have higher spin-flip rates, which wash out the circular-polarization effects observed here.

To show the crucial role of the reservoir excitons, we place a weak, nonresonant, linearly polarized probe beam on top of the condensate, as shown in Fig. 4(a). The probe beam, which has just a small fraction of the four-spot pump power $\left(<0.025 P_{\text {th }}\right)$ induces a reservoir of excitons that overlap with the condensate, but it does not stop the condensation or reduce the condensate density below the critical circular-polarization density. We then measure the absolute average circular-polarization degree in each realization, as a function of the weak probe power. As shown in Fig. 4(b), the circular-polarization degree decreases monotonously as the probe power increases, demonstrating the quenching of circular polarization due to
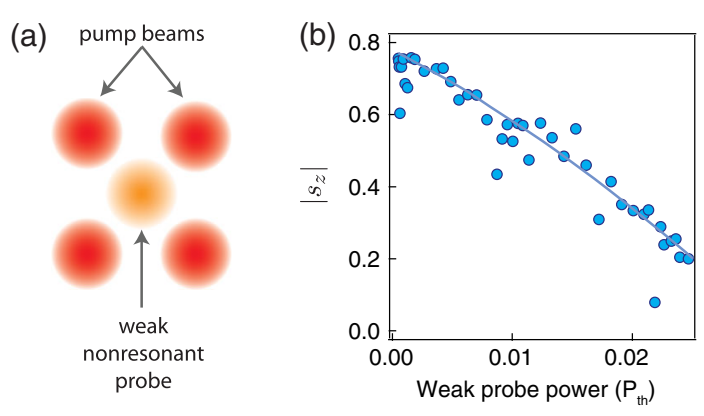

FIG. 4. (a) Nonresonant, linearly polarized, weak probe overlapped with the condensate. (b) Average circular-polarization degree vs power of the probe beam. The blue line is a guide to the eye.

the influence of the overlapping reservoir. This measurement evidences the importance of the separation of the pump-induced reservoir and the trapped condensate in the observation of ferromagnetic condensates.

Four scenarios might explain the buildup of the stochastic circular polarization depending on populations of samespin and opposite-spin (or cross-spin) polaritons: (i) a higher interaction energy for cross-spin than for same-spin polaritons in a condensate which is in thermal equilibrium, (ii) a higher gain from the cross-spin reservoir, (iii) densitydependent losses enhanced by cross-spin condensed polaritons, and (iv) linear polarization energy splitting accompanied by a dissipation rate splitting that destabilizes the linearly polarized condensate ("spin bifurcation"). As we will show now, our experimental data strongly suggest that scenario (iv) is the correct explanation. In the first scenario ("energy minimization"), the condensate free energy is minimized when it acquires circular polarization. This situation could happen in a condensate in thermal equilibrium if the interaction energy of cross-spin polaritons is stronger than that of same-spin polaritons, meaning that the coexistence of cross-polarized polaritons is not favored [41]. However, the interaction with opposite-spin polaritons is well known to be weaker than, and opposite to, that of same-spin polaritons $[29,42,43]$. Moreover, being externally driven and having short lifetimes, polariton condensates are generally far from thermal equilibrium [44]. We note that "energetical" mechanisms cannot explain why states with elliptical polarization (i.e., not fully circular) are formed. In the second scenario ("cross gain"), in order to acquire a circular degree of polarization, the condensate must experience larger gain from the cross-spin reservoir than from the same-spin reservoir. However, the scattering rate from the cross-spin reservoir into the condensate is measured to be significantly smaller than the same-spin reservoir [18]. The third scenario ("cross loss") requires the condensate loss rate to increase when opposite spins are present. If polaritons are directly excited by light, observations have suggested that biexciton formation can produce such enhanced losses [26]. However, this is only 
significant when the relative spectral detuning of the cavity and exciton is small $(<2 \mathrm{meV})$, which is far from the case here. We observe the spontaneous buildup of circular polarization throughout the entire +2 to $-10 \mathrm{meV}$ detuning range. In the remaining scenario (spin bifurcation), which we present in Sec. III for the first time, the buildup of circular polarization is caused by small differences of the energy and dissipation rates of two orthogonal linearly polarized polariton modes, which is present even at $k=0$.

Strain-induced splitting of the linear components of polariton condensates has been demonstrated previously $[45,46]$. In our sample, this splitting varies depending on the position on the sample. We observe a linear polarization energy splitting of up to about $100 \mu \mathrm{eV}$ depending on the position of the excitation on the membranes. Note that all the effects reported here are also seen on unetched samples, so strain from patterning is not crucial. However, we observe a higher splitting at the edges of the membrane than in the middle, as there is more stress in the structure at the edges. Because of the curvature of the cavity stop band, any energy splitting is accompanied by a difference of the linewidth (dissipation rate), as explained in Appendix B. This energy splitting between the two linear components combined with a difference in dissipation rates causes the polarization of the condensate to change from linear polarization to circular at a critical density (see Sec. III).

We emphasize that the stochastic circular polarization here cannot be explained in the framework of the optical spin Hall effect $[28,47]$. In our trapping geometry, the condensate is formed at the ground state with $(\bar{k}=0$, $\delta k=0.4 \mu \mathrm{m}^{-1}$ ) [Fig. 1(c)], where the transverse-electric and transverse-magnetic (TE-TM) splitting vanishes [38]. The trap diameter here is 6 times smaller than the observed spin-ring patterns measured for a nonequilibrium condensate formed at much higher in-plane wave vectors in the same sample [28]. Moreover, the geometry or the orientation of the trap does not affect the polarization state of the condensate. We also see the stochastic circular polarization with a ring-shaped trap and also in high-order spatial mode condensates $[48,49]$. Finally, it should be noted that any theoretical picture that assumes the buildup of circular polarization arises only because of the geometrical arrangement of the pump would necessarily fail to explain the most essential part of this work, which is the spontaneous symmetry breaking (stochastic behavior).

\section{SPIN BIFURCATION THEORY (BROKEN PARITY)}

Our theory is a development of the theory of polariton weak lasing in two coupled condensation centers [16], now for the case of the spin degree of freedom. Here, we have right and left-circular polarizations instead of two separated condensates, and we also allow for the gain-saturation nonlinearity in the system.
The order parameter for an exciton-polariton condensate is a two-component complex vector $\Psi=\left[\psi_{+1}, \psi_{-1}\right]^{\mathrm{T}}$, where $\psi_{+1}$ and $\psi_{-1}$ are the spin-up and spin-down wave functions. The components of the order parameter define the measurable condensate pseudospin $\mathbf{S}=$ $(1 / 2)\left(\Psi^{\dagger} \cdot \boldsymbol{\sigma} \cdot \Psi\right)$, and the normalized spin vector $\hat{\mathbf{s}}=\mathbf{S} / S$, where $\sigma_{x, y, z}$ are the Pauli matrices. The components of this vector contain information about the intensities and relative phases of the emitted light. The order parameter evolves according to the driven dissipative equation

$$
\begin{aligned}
\mathrm{i} \frac{d \Psi}{d t}= & -\frac{\mathrm{i}}{2} g(S) \Psi-\frac{\mathrm{i}}{2}(\gamma-\mathrm{i} \varepsilon) \sigma_{x} \Psi \\
& +\frac{1}{2}\left[\left(\alpha_{1}+\alpha_{2}\right) S+\left(\alpha_{1}-\alpha_{2}\right) S_{z} \sigma_{z}\right] \Psi,
\end{aligned}
$$

or in components,

$$
\begin{aligned}
\dot{\psi}_{+1}= & -\frac{1}{2} g(S) \psi_{+1}-\frac{1}{2}(\gamma-\mathrm{i} \varepsilon) \psi_{-1} \\
& -\frac{\mathrm{i}}{2}\left(\alpha_{1}\left|\psi_{+1}\right|^{2}+\alpha_{2}\left|\psi_{-1}\right|^{2}\right) \psi_{+1}, \\
\dot{\psi}_{-1}= & -\frac{1}{2} g(S) \psi_{-1}-\frac{1}{2}(\gamma-\mathrm{i} \varepsilon) \psi_{+1} \\
& -\frac{\mathrm{i}}{2}\left(\alpha_{1}\left|\psi_{-1}\right|^{2}+\alpha_{2}\left|\psi_{+1}\right|^{2}\right) \psi_{-1} .
\end{aligned}
$$

Here, $g(S)=\Gamma-W+\eta S$ is the pumping-dissipation balance, $\Gamma$ is the (average) dissipation rate, $W$ is the incoherent in-scattering (or "harvest" rate), and $\eta$ captures the gainsaturation term with $S=\left(\left|\psi_{+1}\right|^{2}+\left|\psi_{-1}\right|^{2}\right) / 2$ [50]. This gain saturation depends on the total occupation of the condensate (treated more generally in Appendix C). It is assumed now that $X$ (horizontal) and $Y$ (vertical) linearly polarized singlepolariton states have different energies and dissipation rates. The energy of the $X$-polarized state is shifted by $-\varepsilon / 2$, and the energy of the $Y$-polarized state by $+\varepsilon / 2$. The dissipation rate from the $X$-polarized state is $\Gamma+\gamma$, while the dissipation rate from the $Y$-polarized state is $\Gamma-\gamma$ (see also Appendix B). Finally, $\alpha_{1}$ is the repulsive interaction constant for polaritons with the same spin, and $\alpha_{2}$ is the interaction constant for polaritons with opposite spins.

From Eq. (1), we obtain for the components of the pseudospin vector $\left(\alpha=\alpha_{1}-\alpha_{2}\right)$ :

$$
\begin{aligned}
& \dot{S}_{x}=-g(S) S_{x}-\gamma S-\alpha S_{z} S_{y}, \\
& \dot{S}_{y}=-g(S) S_{y}+\varepsilon S_{z}+\alpha S_{z} S_{x}, \\
& \dot{S}_{z}=-g(S) S_{z}-\varepsilon S_{y},
\end{aligned}
$$

and the related equation for the total spin $\dot{S}=-g(S) S-\gamma S_{x}$. There are two sets of solutions, which we call here the paramagnetic and ferromagnetic solutions. 


\section{A. Paramagnetic solutions}

These give simple condensation into either $X$ or $Y$ linearly polarized states. The $Y$ state possesses the longest lifetime, and the condensation threshold is reached for this state first at $W_{1}=\Gamma-\gamma$. There is no parity breaking for this condensate: $S_{y}=S_{z}=0, \quad S_{x}=-S$ with $S=\left(W-W_{1}\right) / \eta$, so that the occupations of +1 and -1 components are equal. However, this condensate solution becomes unstable for $W>W_{2}$. The values of the critical occupation $S_{c}$ and the critical pumping rate are

$$
S_{c}=\frac{\gamma^{2}+\varepsilon^{2}}{\alpha \varepsilon}, \quad W_{2}=W_{1}+\eta S_{c} .
$$

Note that this instability is present also for equal dissipation rates, i.e., when $\gamma=0$. In this case, the system [Eqs. (3a)-3(c)] describes the self-induced Larmor precession of the pseudospin vector. Incorporating energy relaxation (e.g., using small negative $\gamma$ ) then leads to the formation of the $X$-polarized condensate-an intuitively expected result.

\section{B. Ferromagnetic solutions}

The key ingredient of our theory is the presence of the $\gamma>0$ parameter describing the variation of dissipation rates. This parameter allows the formation of the "weak lasing" regime [16], which is characterized by two important features: (i) The $X$-polarized condensate is also unstable, and (ii) when the $Y$-polarized condensate loses stability at the critical occupation $S_{c}$, it continuously transforms into one of the two ferromagnetic states. While Eqs. (2) are parity symmetric, i.e., they are not affected by the interchange of left- and right-circular polarization, the new solutions are characterized by broken-parity symmetry and by spontaneous formation of either left or right elliptical polarization. These solutions are
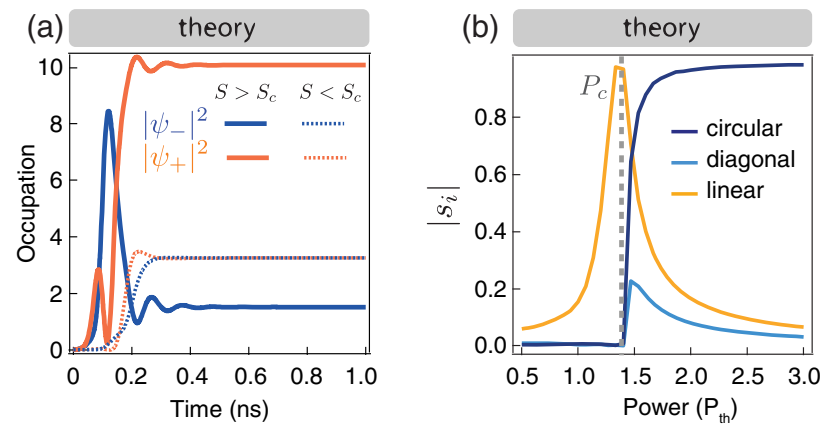

$S_{z}= \pm \frac{\varepsilon}{\gamma} \sqrt{\frac{\gamma^{2}-g(S)^{2}}{\varepsilon^{2}+g(S)^{2}}} S, \quad S=\frac{\gamma\left[\varepsilon^{2}+g(S)^{2}\right]}{\alpha \varepsilon g(S)}$,

where the positive root of the second formula in Eq. (5b) should be taken. We note that while the sign of $S_{x}$ is always negative, $S_{y}$ and $S_{z}$ have opposite signs for the two solutions. This means that the left-circular component is accompanied by a diagonal component, and the rightcircular component by an antidiagonal component. Moreover, if these components change for some reason, they mirror each other as long as the total condensate occupation stays fixed. We label these two solutions as the $s_{\downarrow}$ and $s_{\uparrow}$ spin states.

The spin-independent model for the gain saturation used in this section is sufficient to describe the experimentally observed features. However, the spin relaxation in the reservoir can be slow [40], and in this case, the model should be modified to allow the saturation terms to depend on the individual occupations of the left- and the rightcircular-polarization components rather than the total occupation only. The parity breaking is still present after this modification; however, the stability of solutions becomes more complex. The ferromagnetic solutions can now become unstable and transform into periodic cycles [51]; the dynamics of the pseudospin can become irregular, and this can also result in the formation of the stable $X$-polarized condensate at high pumping powers. See Appendix $\mathrm{C}$ for more details.

Numerical calculations for the occupation of the two circular components of the wave function when $S<S_{c}$ (dotted lines) and when $S>S_{c}$ are shown in Fig. 5(a). Here, the condensate is initialized with a small asymmetry in spin-up and spin-down occupations $(<1 \%)$. Below the critical occupation $S_{c}$, the condensate is linearly polarized, but when the occupation is increased above the threshold
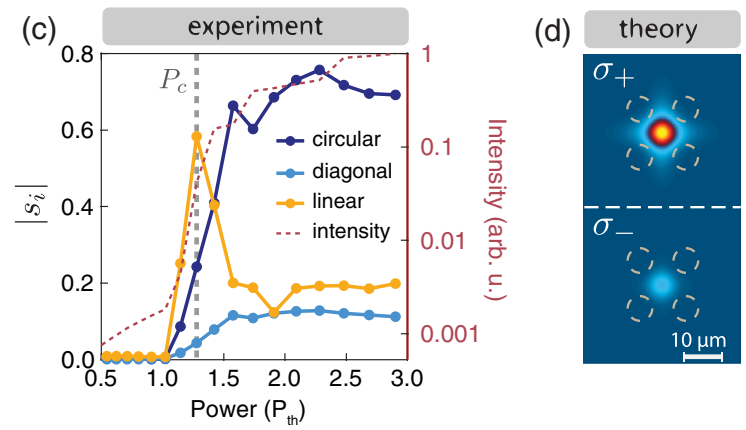

FIG. 5. (a) Numerical calculations using Eq. (3) for the case when the condensate occupation is below the critical occupation $S_{c}$ (dotted lines) and when it is above $S_{c}$ (solid lines). Blue lines show the occupation of the spin-down component, and orange lines show the spin-up component. (b) Numerical calculations of the Stokes parameters at different powers using Eq. (3). (c) Measured power dependence of the condensate Stokes parameters. (d) 2D simulations, with asymmetric initial conditions $\left[s_{z}(t=0)<0.01\right]$, show density of the $\psi_{ \pm}$components of the wave function. Pump spots are marked by dashed lines (see Appendix G for parameters). 
$S_{c}$, the condensate adopts one of two elliptically polarized configurations depending on the initial conditions. In the experiment, the stochastic behavior is due to random spin fluctuations at the onset of the condensation. In theory, we can reproduce it by randomly setting the initial conditions. Numerical calculations of the condensate polarization versus excitation power are shown in Fig. 5(b). Directly at the condensation threshold, the condensate is linearly polarized, but once it reaches the critical occupation (at $P_{c}=1.3 P_{\text {th }}$, marked by a dashed grey line), the linear component is quenched and circular polarization builds up. This behavior reproduces the experimental data, as shown in Fig. 5(c). We observe an initial buildup of linear polarization at $P_{\text {th }}=25 \mathrm{~mW}$, and subsequent quenching of it with the continuing increase of circular polarization at $P_{c}=1.25 P_{\text {th }}$ (marked by a dashed grey line, with total intensity marked by a dotted red line). Once strong degree of circular polarization is achieved, the orientation of the condensate circular polarization becomes stochastic under linearly polarized pumping.

We can extend Eq. (1) and account for 2D real-space degrees of freedom by using complex Ginzburg-Landautype equations $[8,35,50]$, which in addition to the pump and decay also incorporate a repulsive potential due to the excitons in the pump spots and an energy relaxation [52] for polaritons in the trap:

$$
\begin{aligned}
\mathrm{i} \frac{d \Psi}{d t}= & -\frac{\mathrm{i}}{2}\left[g(S)+\gamma \sigma_{x}\right] \Psi \\
& +(1-\mathrm{i} \Lambda)\left\{\frac{1}{2}\left[\left(\alpha_{1}+\alpha_{2}\right) S+\left(\alpha_{1}-\alpha_{2}\right) S_{z} \sigma_{z}\right] \Psi\right. \\
& \left.-\frac{1}{2} \varepsilon \sigma_{x} \Psi-\frac{\nabla^{2}}{2 m^{*}} \Psi+V_{p} \Psi\right\},
\end{aligned}
$$

where $m^{*}$ is the effective mass of the polaritons. The harvest rate is given by $W=r P / \Gamma_{R}$, where $P$ is the spinindependent spatial profile of the excitation, $\Gamma_{R}$ is the decay rate of the exciton reservoir, and $r$ is the incoming rate of polaritons into the condensate. The gain saturation is given by $\eta=r^{2} P / \Gamma_{R}^{2}$. The repulsive potential due to the interaction of polaritons with the exciton reservoir is given by $V_{p}=\frac{1}{2} g_{r} N+\frac{1}{2} g_{P} P$, where $g_{r}$ and $g_{P}$ are the interaction constants of polaritons with the exciton reservoir and the pump spot, respectively, and $N=g(S) / r$ is the density of the exciton reservoir. Here, $\Lambda \ll 1$ is a phenomenological constant that gives the energy relaxation. The density profile of the two circular components of the wave function in the steady state [Fig. 5(d)], for the case of a trapped condensate in the middle of the four pump spots, exhibits a circular-polarization degree of $\left|s_{z}\right|=0.69$. Note that with $\gamma=0$ and only polarization splitting (including TE-TM splitting), our 2D simulations do not show bistable condensation.
It is important to note the differences between the ferromagnetic states we discuss here and the magnetization transition in equilibrium cold atom systems $[53,54]$. First, the parity-breaking bifurcation described above does not reduce the energy of the system (unlike for atoms). In fact, the energy of elliptically polarized states is higher than that of linearly polarized states. Second, the in-plane components of the spin do not vanish completely. Third, we have a magnetized condensate with only two possible orientations, whereas in atomic systems, ferromagnetic domains with continuous variable orientation are observed.

If the Hamiltonian and the initial state of a system are symmetric under the exchange of spin-up and spin-down components, but the final state is not, the parity symmetry is spontaneously broken. This is indeed the case here: We excite an equal population of spin-up and spin-down polaritons, which spontaneously condense but form highly circularly polarized macroscopic states in the absence of any external magnetic field.

\section{RESONANT EXCITATION}

The GaAs substrate commonly used in the fabrication of GaAs microcavities is opaque at the emission wavelength (around $800 \mathrm{~nm}$ ) of the cavity polaritons. As a result, the back side of the cavity is resonantly inaccessible. Resonant excitation of the cavity from the front side has complications with backscatter from the laser, especially in high finesse cavities and at normal incidence, where the condensate emission mode is located. To circumvent this problem, we chemically etch the substrate to form membranes of $8.8 \mu \mathrm{m}$ thickness and $300 \mu \mathrm{m}$ diameter [see Fig. 1(a)]. For resonant excitation, we use a narrowlinewidth $(<2 \mathrm{GHz}) \mathrm{CW}$ laser, which is amplitude modulated with a second AOM. We call this resonant laser the "gate." We use two photomultipliers and a fast oscilloscope to time resolve the left- $\left(\sigma_{-}\right)$and right-circular $\left(\sigma_{+}\right)$ polarization intensity of the condensate emission. The resonant excitation laser, the nonresonant pump laser, the cameras, and the oscilloscope are all synchronized, which allows us to vary the delay time and amplitudes of each laser pulse on demand.

\section{A. Resonant initialization of spin states}

We can control the polarization of the trapped condensate with the gate laser. In this case, we additionally resonantly excite the condensate (which is generated by the four nonresonant pump spots) with a second laser from the backside of the microcavity membrane. This gate can be linearly, left-, or right-circularly polarized. Figure 6 shows the condensate circular degree of polarization versus that of the pump. In the green curve, which shows the behavior when the gate is linearly polarized, we reproduce the same result as that in Fig. 3(d). However, when a right (or left) circularly polarized gate is applied, the curve shifts to the 


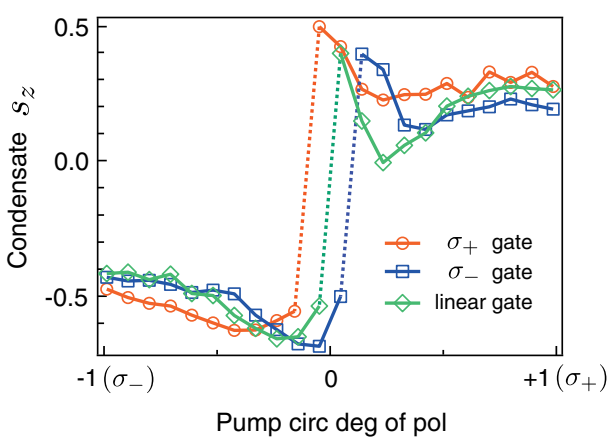

FIG. 6. Measured circular polarization of condensate vs pump ellipticity, for three different gate polarizations. Dashed lines mark the region where the condensate initializes stochastically in spin-up or spin-down states in each realization.

left (or right). In other words, with a linearly polarized pump, the condensate initializes in a right (or left) circularly polarized state. The imbalance caused by the resonant gate cancels out with an opposite circularly polarized pump with a circular-polarization degree of $\left|s_{z, \text { pump }}\right| \sim 0.1$.

\section{B. Elliptically polarized pump: Coherent driving}

In a first experiment, we initialize a condensate in the spin-down state $\left(s_{\downarrow}\right)$ by making the pump laser slightly leftcircularly polarized ("asymmetric pumping"). Once the condensate is created, we excite the sample resonantly from the back side with an oppositely circularly polarized $\left(\sigma_{+}\right)$ gate. Figure 7(a) shows the intensity of $\sigma_{+}$and $\sigma_{-}$ components of the condensate emission during this gating. We see a reduction in intensity for the component, which is opposite to the gate laser polarization, and an increase for the same spin polarization component. To account for this in the theory, we add a new term to Eq. (2), corresponding to the resonant laser:

$$
\begin{aligned}
\dot{\psi}_{+1}= & -\frac{1}{2} g_{+1}(S) \psi_{+1}-\frac{1}{2}(\gamma-\mathrm{i} \varepsilon) \psi_{-1} \\
& -\frac{\mathrm{i}}{2}\left(\alpha_{1}\left|\psi_{+1}\right|^{2}+\alpha_{2}\left|\psi_{-1}\right|^{2}\right) \psi_{+1} \\
& -\mathrm{i} A \Pi\left(t, t_{0}, \delta t\right) e^{-\mathrm{i} \omega_{g} t},
\end{aligned}
$$

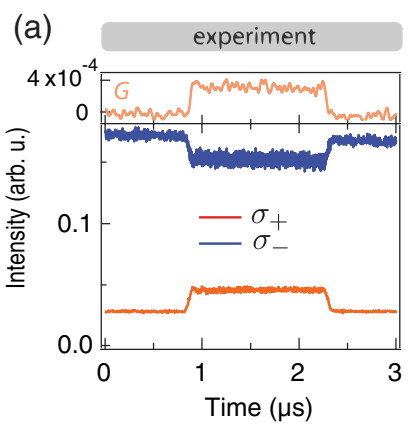

(d)

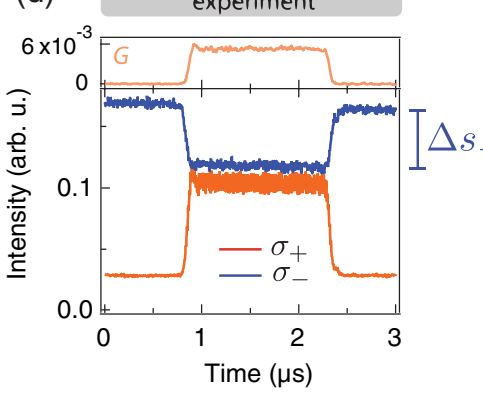

(b)

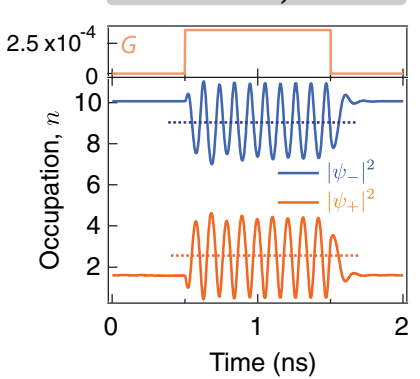

(e)

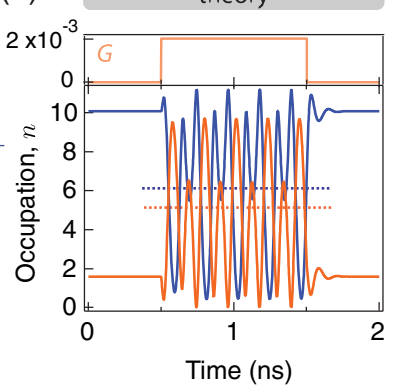

(c)

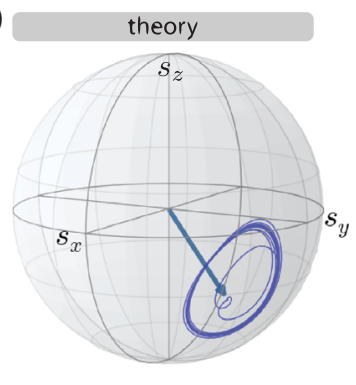

(f)

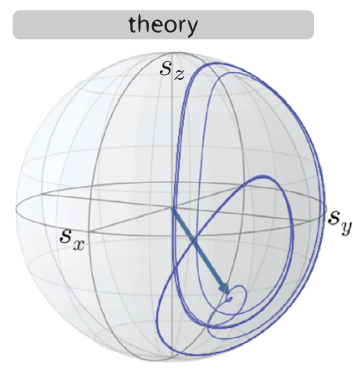

(g)

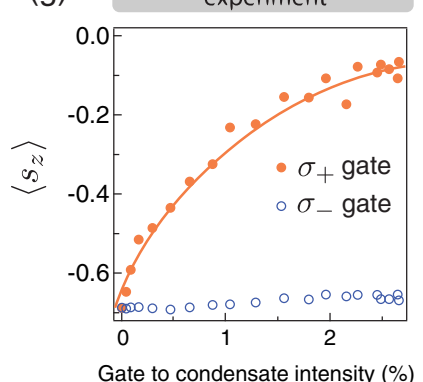

(h)

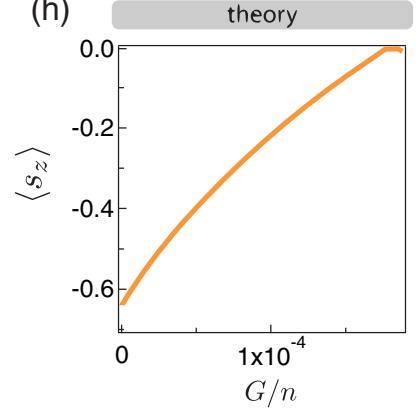

FIG. 7. (a) Measured intensity of circular-polarization components $\left(\sigma_{ \pm}\right)$for adding gate pulse $G$ (intensity shown on above inset). The condensate initializes in the $s_{\downarrow}$ state because of the asymmetric pumping condition $s_{z \text {,pump }}=-0.1$. A gate pulse with an opposite circular polarization $\left(\sigma_{+}\right)$is applied. (b) Numerical calculations for (a), showing that during the gate pulse $G=A^{2}$ the spin-up and spin-down densities oscillate with a $\pi$ phase shift. (c) Time evolution of the pseudospin, showing how the pseudospin orbits around the spin-down stationary state (marked by the blue arrow) when the gate pulse is applied. (d) Same as in (a) but with a fivefold increase in the intensity of the gate. The time-averaged condensate emission is almost circularly unpolarized when the pulse is applied $\left(\left\langle s_{z}\right\rangle=0\right)$. (e) Numerical calculations for (d), showing almost equal amplitude oscillation of the spin-up and spin-down densities. (f) Trajectory of pseudospin, showing that with a strong gate pulse, large orbits commence around the two spin-up and spin-down stationary states. (g) Average circular polarization when the gate is on, at different gate intensities. When an opposite polarization gate is applied, $\left\langle s_{z}\right\rangle$ converges to zero, while it remains unchanged for the same-spin gate. The orange line is a guide to the eye. (h) Numerical calculations of $\left\langle s_{z}\right\rangle$ vs normalized pump intensity ( $n$ is the condensate occupation). 


$$
\begin{aligned}
\dot{\psi}_{-1}= & -\frac{1}{2} g_{-1}(S) \psi_{-1}-\frac{1}{2}(\gamma-\mathrm{i} \varepsilon) \psi_{+1} \\
& -\frac{\mathrm{i}}{2}\left(\alpha_{1}\left|\psi_{-1}\right|^{2}+\alpha_{2}\left|\psi_{+1}\right|^{2}\right) \psi_{-1},
\end{aligned}
$$

where $A \Pi\left(t, t_{0}, \delta t\right)=A H\left(t-t_{0}\right) H\left(t_{0}+\delta t-t\right)$ uses the Heaviside step function $H$ to give a square pulse with amplitude $A$, which starts at time $t_{0}$ and lasts for $\delta t$, and $\omega_{g}$ is the excitation frequency. To account for the elliptically polarized pumping, we modify $g(S)$ to $g_{ \pm 1}(S)=$ $\Gamma-W_{ \pm 1}+\eta S$. Numerical calculations [Fig. 7(b)] show the case $W_{-1}=1.09 W_{+1}, A=5 \times 10^{-4} n$, where $n$ is the occupation of the condensate, and $\omega_{g}=-0.3 \varepsilon$. The dotted line marks the time average of the oscillations. The condensate pseudospin vector precesses around the stationary state pseudospin vector at a frequency of $\omega_{L} / 2 \pi \sim$ $10 \mathrm{GHz}$ in a limit cycle [see Fig. 7(c)]. In the case where the condensate is highly spin polarized and the gate is at resonance $\left(\omega_{g}=0\right)$, the oscillation frequency is equal to the self-induced Larmor precession frequency $\omega_{L}=\gamma \varepsilon / g$ (Appendix F). This case sets the fastest possible spin dynamics in the system and does not depend on the gate intensity. Here, the condensate pseudospin oscillates faster than our detection time resolution, and consequently, we only see the average effect in Fig. 7(a). The coherent driving reported here strongly depends on the detuning of the gate laser frequency relative to that of the condensate. The resonance width at which we can drive the condensate is determined to be $10-20 \mu \mathrm{eV}$, as explained in Appendix E.

In the case of Fig. 7(d), the intensity of the gate laser is increased by 5 times with respect to that in (a). Under these conditions, the time average of the circular component of the condensate emission becomes almost unpolarized. If the amplitude of the resonant excitation is large enough, the condensate pseudospin can cross over to the second attractor (the spin-up state) and form a large trajectory that encompasses both spin-up and spin-down stationary states with a characteristic period doubling [55] [Figs. 7(e) and 7(f))]. The dotted lines in (e) mark the time average of the oscillations. Figure $7(\mathrm{~g})$ shows the power dependence of the average circular polarization as a function of the intensity of the gate. As the oppositely polarized gate intensity is increased, we see the condensate average circular polarization converges to zero. Here, we have the gate resonant with the condensate. However, the coherent driving strongly depends on the detuning of the gate laser frequency to that of the condensate. The resonance width where we can drive the condensate is determined to be $10-20 \mu \mathrm{eV}$, as explained in Appendix E. That theory and experiment agree regarding the observed average polarization over the whole range of gate powers, and also the observation of a resonance (see Appendix E) strongly suggests that our description in terms of coherent driving is valid.

In summary, the fixed points of broken-parity symmetry $\left(s_{\downarrow}, s_{\uparrow}\right)$ become unstable because of small perturbations and convert to limit cycles around stationary states. The precession is linear for small gate amplitudes, but it becomes nonlinear for large amplitudes. The limit cycle is manifested by oscillation of the condensate parameters (occupation and polarization) with time. In the linear regime, the gate pulse appears to act as if it induces an effective magnetic field, around which the condensate polarization precesses. It is worth noting that this system can exhibit chaotic behavior with increased amplitude of the pulse. Increasing the amplitude first results in period doubling and eventually leads to chaos (Feigenbaum scenario) [55]. The ability to coherently control the spin
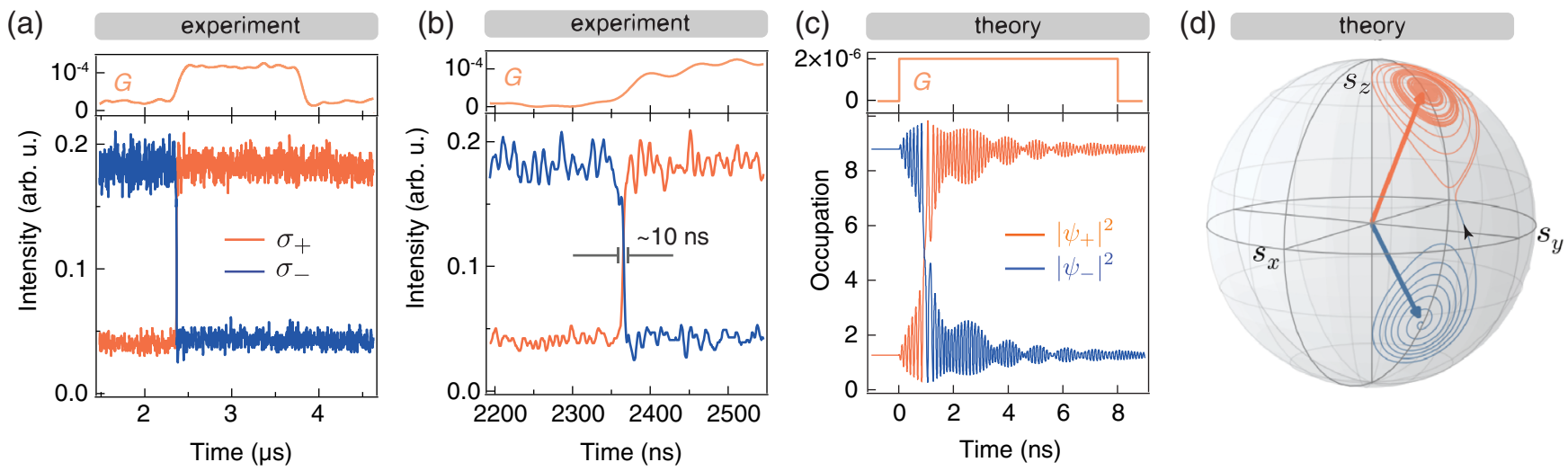

FIG. 8. (a) Intensity of the spin-up and spin-down states as a function of time. Resonant pulse $G$, with opposite circular polarization $\left(\sigma_{+}\right)$, switches the circular polarization of the condensate. (b) Despite the slow rise time of our AOM (around 100 ns), the condensate switches to the opposite spin state in less than $10 \mathrm{~ns}$ (the limit of our detection), as soon as the intensity crosses the spin-switching threshold. (c) Numerical calculations for (a), showing that the spin switches with a gate pulse with intensity 6 orders of magnitude smaller than that of the condensate. The evolution of the pseudospin is shown in (d). The two attractors, marked by the blue and orange arrows, are the two stationary $s_{\uparrow \downarrow}$ spin-polarized states. 
of the condensate suggests its utility for computational operations.

\section{Linearly polarized pump: Spin switching}

We now explain the case where the nonresonant pump is linearly polarized. In this case, there is no external "force" to drive the condensate to a specific spin state. As a result, once the circular component of the condensate pseudospin crosses zero, it falls into the opposite spin state's attractor. Figure 8(a) shows a realization that lasts for $4 \mu$ s with the condensate first initialized in the spin-down state $\left(s_{\downarrow}\right)$. We resonantly excite the condensate with an oppositely polarized gate $\left(\sigma_{+}\right)$. This excitation causes the switching of the polarization of the condensate to the same polarization as that of the gate. The gate, which is $1.5 \mu \mathrm{s}$ in duration, is then turned off, but the condensate remains in the switched polarization because the pump is linearly polarized and the symmetry is not explicitly broken. Therefore, we are capable of manipulating the polarization of the condensate on demand, while also reinforcing our observation that the condensate picks a polarization spontaneously when it is formed. It should be noted that the state of the condensate does not change when the circular polarization of the gate is the same as that of the condensate.

If the symmetry breaking was somehow set by the parameters of the experiment, as in the previous example where the pump was slightly elliptical, the condensate would switch back to its original state after the gate beam was turned off. Instead, a very small gate field in the cavity can initiate coherent switching. The upper panel in Fig. 8(a) shows the transmitted gate intensity when the pump is blocked. Indeed, the gate power before the cavity is $600 \mu \mathrm{W}$, which is 50 times weaker than the pump power. However, only a small fraction of this resonant beam couples to the cavity in our setup. Therefore, a suitable comparison of the intensity of the gate to that of the condensate is to compare the transmitted intensity of the gate to the intensity of the condensate in the same direction. We find that the gate intensity is more than 60 times weaker than the condensate intensity, when the gate laser frequency is tuned to the bottom of the polariton dispersion (accounting for the blueshift of the condensate).

By estimating the occupation number of the condensate (see Appendix D), we find that only 13 polaritons are enough to reverse the spin state of the condensate. The condensate switching time is less than $10 \mathrm{~ns}$ (our detection limit) and is 10 times faster than the switching time of the AOM [Fig. 8(b)]. This important fact shows that the condensate does not follow the optical gate adiabatically. Simulations of the spin switching with minimum resonant gate intensity are shown in Fig. 8(c). The polarization of the gate is opposite to that of the condensate, and we have assumed symmetric pumping rates for the spin-up and spindown condensates, i.e., $W_{+1}=W_{-1}$. The condensate polarization reverses within about 200 ps once the gate is applied, and it remains in that state after the pulse is turned off during continued spin evolution [Fig. 8(d)]. Switching requires a minimum gate intensity to twist the condensate pseudospin onto the equator in the Poincare sphere. This minimum sets a threshold for the gate power. By measuring the gate flux and using the switching time of $10 \mathrm{~ns}$, an upper bound of the minimum energy for switching the state of the condensate is found to be about $1 \times 10^{-15} \mathrm{~J}$, comparable to state-of-the-art optoelectronic switches with similar speeds [56]. We emphasise, however, that the theoretical limit for the minimum switching energy is 50 times smaller because the spin dynamics of the system (around 200 ps) is 50 times faster than our detection limit. This polaritonic system is then an extremely low-power switch.

Multistability has indeed been demonstrated before in resonantly pumped condensates $[11,26]$. However, there are several major differences between our system and that of the Deveaud group. In Deveaud's experiments, the physical process causing multistability is the nonlinear nonradiative losses in the polariton gas due to the formation of biexcitons. These losses are only significant when the polariton gas energy is close to the biexciton energy $(<2 \mathrm{meV})$ [57]. In contrast, here the bistability that we observe is present $>10 \mathrm{meV}$ below the biexciton energy. There is no phase transition in the work of Paraïso et al. [26] on multistability, and symmetry is not spontaneously broken. In contrast, we observe spin symmetry breaking while the power thresholds of the left- and right-circularly polarized states are the same, allowing us to observe spontaneous magnetization. In the Deveaud multistable system, in order to switch the polarization, one has to inject an opposite-spin polariton density equal to the density difference of spin-up and spin-down polaritons. In contrast, in our experiments, the condensate switches with a gate intensity 60 times weaker than the condensate. Theoretically, the gate intensity can be much weaker, and the reason that we do not see switching at even weaker powers experimentally is because of spin fluctuations in the condensate, as discussed in the next subsection. Finally, we note that other microstructured systems such as microdisk lasers can also show bistability. The whispering gallery modes in microdisk lasers can have clockwise or counterclockwise propagating lasing modes [58]. When the coupling between the two modes is small, a cross-gain saturation causes one of the modes to dominate. The system can therefore operate in "flip-flop" mode, and the two modes can be excited and switched with weak optical pulses [59].

\section{Thermal noise: Spin flipping}

Above the spin bifurcation occupation threshold $\left(S_{c}\right)$, any small perturbation induces a "restoring force," which drives the condensate toward the attractor once the perturbation is stopped. This restoring force keeps the condensate around the attractor for small spin fluctuations. However, 

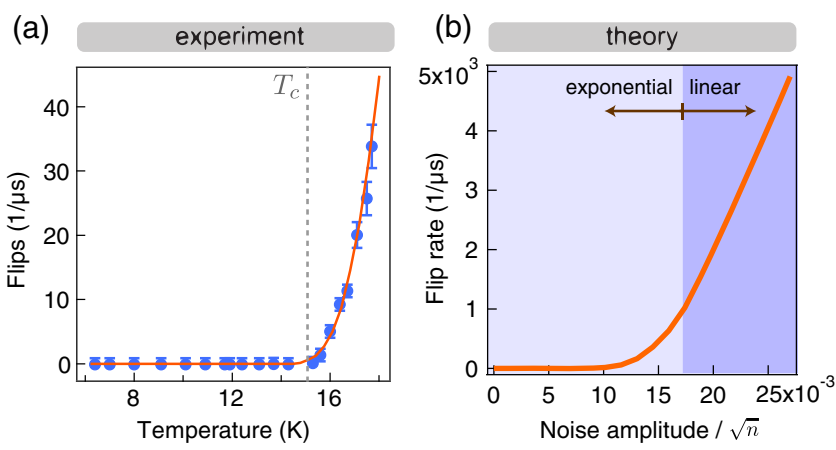

FIG. 9. (a) Measured spontaneous spin-flip rate at different temperatures of the sample. Above the temperature $T_{c} \simeq 15 \mathrm{~K}$, the flip rate increases exponentially. The solid line shows the theoretical fit. (b) Calculated dependence of flip rate with normalized noise amplitude. The flip rate increases exponentially as the noise amplitude increases (light region), and it eventually becomes linear (dark region). Comparison with (a) shows that the measured flip rates remain below this linear regime.

for sufficiently large perturbations, the condensate can flip to the other attractor with the opposite circular polarization. In the experiment, for sample temperatures near $5 \mathrm{~K}$ the observed spin-polarized states remain stable for many seconds (longer than the stability of our experiment can be maintained). However, increasing the temperature above $T_{c} \simeq 15 \mathrm{~K} \pm 1$ induces spin flips in the condensate during a measurement time window of $t_{m}=1.5 \mathrm{~ms}$. The measured rate of the spin flipping beyond $T_{c}$ is a nonlinear function of the sample temperature, as shown in Fig. 9(a). It is important to note that we do not have a real threshold for the occurrence of spin flipping here. In fact, the spin-flip rate increases exponentially with temperature from $T=0$ until it becomes significant in the finite-time measurement window. We can account for this phenomenon by adding a thermally induced noise to our theory. This thermal noise $f(t)$, which is similar to the Johnson noise, is included in Eq. (2) to give Langevin-type equations:

$$
\begin{aligned}
\dot{\psi}_{+1}= & -\frac{1}{2} g(S) \psi_{+1}-\frac{1}{2}(\gamma-\mathrm{i} \varepsilon) \psi_{-1} \\
& -\frac{\mathrm{i}}{2}\left(\alpha_{1}\left|\psi_{+1}\right|^{2}+\alpha_{2}\left|\psi_{-1}\right|^{2}\right) \psi_{+1}+f_{+1}(t), \\
\dot{\psi}_{-1}= & -\frac{1}{2} g(S) \psi_{-1}-\frac{1}{2}(\gamma-\mathrm{i} \varepsilon) \psi_{+1} \\
& -\frac{\mathrm{i}}{2}\left(\alpha_{1}\left|\psi_{-1}\right|^{2}+\alpha_{2}\left|\psi_{+1}\right|^{2}\right) \psi_{-1}+f_{-1}(t),
\end{aligned}
$$

where $f_{\sigma}(t)$ with $\sigma= \pm 1$ is a realization of Gaussian random processes with zero mean $\left\langle f_{\sigma}(t)\right\rangle=0$ and a $\delta$-like two-point correlation function

$$
\left\langle f_{\sigma}(t) f_{\sigma^{\prime}}\left(t^{\prime}\right)\right\rangle=0, \quad\left\langle f_{\sigma}(t) f_{\sigma^{\prime}}^{*}\left(t^{\prime}\right)\right\rangle=2 D \delta_{\sigma, \sigma^{\prime}} \delta\left(t-t^{\prime}\right) .
$$

At finite temperature $T$, the intensity of the noise can be written approximately as $D=\frac{1}{2}(W+a T)$, with the $W$ contribution being a shot noise from the reservoir [16] and the thermal part $a T$ defined by spin-flip polariton-phonon scattering.

The flip rate vs noise amplitude using stochastic simulations [Fig. 9(b)] reveals an Arrhenius-like increase at a critical threshold followed by a crossover to a linear regime. This model gives an excellent account of the dynamics [Fig. 9(a)]. For temperatures beyond $18 \mathrm{~K}$, we reach the time resolution of our detection. As a result, we cannot completely span the crossover to the linear spin-flip regime in our experiment. A fit of the simulation results to the experimental data in Fig. 9(a) gives $a=0.17 \mathrm{ps}^{-1} \mathrm{~K}^{-1}$, which sets the dependence of the spin-flip rate on $D(T)$. We can then explore how this noise perturbs the spin system given by Eqs. (8).

Inclusion of noise produces a spin-flip rate that can overcome the effective spin potential barrier (see Appendix F). For the case in which the circular degree of polarization is high, the spin-flip process can be considered as a one-dimensional Kramers transition. The spin-flip rate $R_{K}$ can then be estimated as

$$
R_{K}=\frac{\sqrt{\varepsilon g(S)}}{2 \pi} \exp \left\{-\frac{g(S) n}{4 D} \ln \left(\frac{\varepsilon}{g(S)}\right)\right\} .
$$

We note that for $\varepsilon=30 \mu \mathrm{eV}$ and the condensate occupation $n=800$, the zero-temperature shot-noise spin-flip rate (set by $W$ ) is negligible for our observation time scales $(10 \mathrm{~s})$. The critical temperature $T_{c}\left(t_{m}\right)$ given by $R_{K}\left(T_{c}\right)=$ $1 / t_{m}$ depends dramatically on the measurement window time $t_{m}$. While the phonon-polariton interaction $a$ in our system gives $T_{c}(1.5 \mathrm{~ms})=15 \mathrm{~K}$, at lower temperatures the condensate spin lifetime rapidly exceeds the stability time of our experimental apparatus (many seconds). Modifying the phonon-polariton interaction $a$ thus has an enormous effect on the spin stability. Finally, we note that in a similar fashion to thermal noise, an overlapping reservoir can also induce spin noise in the condensate. The spin noise causes condensate spin flips, which result in the reduction of the time-averaged circular polarization. Theoretically, this can be studied by introducing a noise term similar to Eq. (9), but instead of depending on temperature, the noise intensity depends on the overlapping reservoir density [60].

\section{CONCLUDING REMARKS}

In summary, we showed how spin can emerge spontaneously in nonresonantly pumped polariton condensates. We found that for trapped condensates, in the case where the pump is linearly polarized, parity symmetry is spontaneously broken by spin fluctuations at the onset of condensation. Fluctuations are amplified by nonlinearities 
in the condensate formation due to the energy and lifetime splitting of the linear polarization components, producing a spin-up or spin-down condensate. The symmetry can be explicitly broken by applying a slightly elliptically polarized pump, which increases the likelihood of forming condensates with the same spin as the pump. In the case where the pump is linearly polarized, we switched the condensate state using a 60 -fold weaker resonant gate pulse with an opposite circular polarization. This situation changes when the pump is elliptically polarized, where instead of switching, the condensate pseudospin precesses around stationary states in limit cycles. Finally, we showed how thermal excitations can induce spin flips with a rate that increases exponentially with sample temperature.

We demonstrated here one way to explicitly break symmetry utilizing elliptical polarization pumping geometries. One could also break the symmetry by introducing a magnetic field to split the energy of the spin-down and spin-up condensates. Alternatively, the pumping symmetry could be broken with spin-current injection. These experiments thus exhibit rich physics with potential applications in sensing.

The observation of spontaneous discretized spinpolarized states also has interesting consequences in the physics of condensate lattices. The possibility of shared reservoirs, and a Josephson-type tunneling [61] between adjacent sites, could provide new phenomena previously unobserved in driven bosonic systems. Magnetic phase transitions, geometric frustration, and spontaneous pattern formation of spin in lattices, domain formation, topological spin insulators, and topological defects are a few examples of magnetic systems that could be studied, all within a highly controlled bosonic many-body system.

While the condensed polariton lattice resembles nanomagnet arrays $[62,63]$, it has the inherent advantages of tunable nonlinearity, longer spin relaxation time, ps response, rapid optical addressing and manipulation, and adaptable scalability. The spin-polarized state at zero magnetic field is retained for many seconds, 10 orders of magnitude longer than the condensation time, making it a suitable candidate for optical spin-based memories.

Spin switching with only a fraction of the condensate density, which is a direct result of the nonlinearity in our system, can be used for low-power optical and electrical sensing and spin switches. Finally, the possibility of coherent driving allows the realization of superpositions of spin-up and spin-down states, which are the key requirements for quantum information processing.

\section{ACKNOWLEDGMENTS}

We acknowledge discussions with B. L. Altshuler and N. G. Berloff. This work was supported by EPSRC Grant No. EP/G060649/1, EP/L027151/1, EU Grant No. CLERMONT4 235114, EU Grant No. INDEX 289968, ERC Grant No. LINASS 320503, the
Leverhulme Trust Grant No. VP1-2013-011, Spanish MEC (Grant No. MAT2008-01555), the Greek GSRT ARISTEIA Apollo program and Fundación La Caixa, and Mexican CONACYT Grant No. 251808. F. P. acknowledges financial support from EPSRC at the University of Cambridge and a Schrödinger grant at the University of Oxford. The data corresponding to all the figures in this paper can be found at https://www.repository.cam.ac.uk/ handle/1810/248036.

\section{APPENDIX A: EXPERIMENTAL METHODS}

The cavity's top (bottom) distributed Bragg reflector (DBR) is made of 32 (35) pairs of $\mathrm{Al}_{0.15} \mathrm{Ga}_{0.85} \mathrm{As} / \mathrm{AlAs}$ layers of $57.2 \mathrm{~nm} / 65.4 \mathrm{~nm}$. Four sets of three $10-\mathrm{nm} \mathrm{GaAs}$ quantum wells (QW) separated by 10-nm-thick layers of $\mathrm{Al}_{0.3} \mathrm{Ga}_{0.7} \mathrm{As}$ are placed at the maxima of the cavity light field. The $5 \lambda / 2(583-\mathrm{nm})$ cavity is made of $\mathrm{Al}_{0.3} \mathrm{Ga}_{0.7} \mathrm{As}$. The microcavity sample is chemically etched from the substrate side to form 300- $\mu$ m-diameter membranes, allowing optical access from the back of the sample for resonant excitation [Fig. 1(a)]. The sample shows condensation under nonresonant excitation [64]. The excitation laser is a single-mode CW Ti:Sapphire, which is amplitude modulated using an AOM with a rise time of $100 \mathrm{~ns}$. To pattern the pump intensity, a spatial light modulator was used [48].

\section{APPENDIX B: STRAIN-INDUCED LINEAR POLARIZATION SPLITTING}

The measured energy of the ground-state $X$ - and $Y$ polarized photoluminescence far below threshold is shown in Fig. 10(a). The energy splitting of the linearly polarized modes varies across the sample surface, reaching its maximum at the edges and minimum at the center of the membranes. This observation suggests that the splitting is correlated to the level of strain across the microcavity structure, since the latter is expected to possess a similar spatial dependence: namely, to be strongest at the boundary between etched and nonetched regions and relax towards the central parts of the membranes. Note that we observe all the same phenomena even in unetched samples, as strain is universally present from the III-V heterostructure growth.

Strain-induced splitting of the initially degenerate polariton states at $k=0$ into orthogonal linearly polarized modes has been demonstrated in previous works $[45,46]$. Both the excitonic and the photonic parts of the polariton can be affected by strain to produce such an anisotropy. In the former case, the initial splitting of the bright exciton states due to exchange interactions is enhanced by straininduced mixing of the heavy and light hole valence bands, thereby reducing the symmetry of the QW $[45,65,66]$. In the latter case, strain induces a small birefringence in the cavity and/or DBRs, hence lifting the degeneracy between the [110] and [110] axes [46]. 

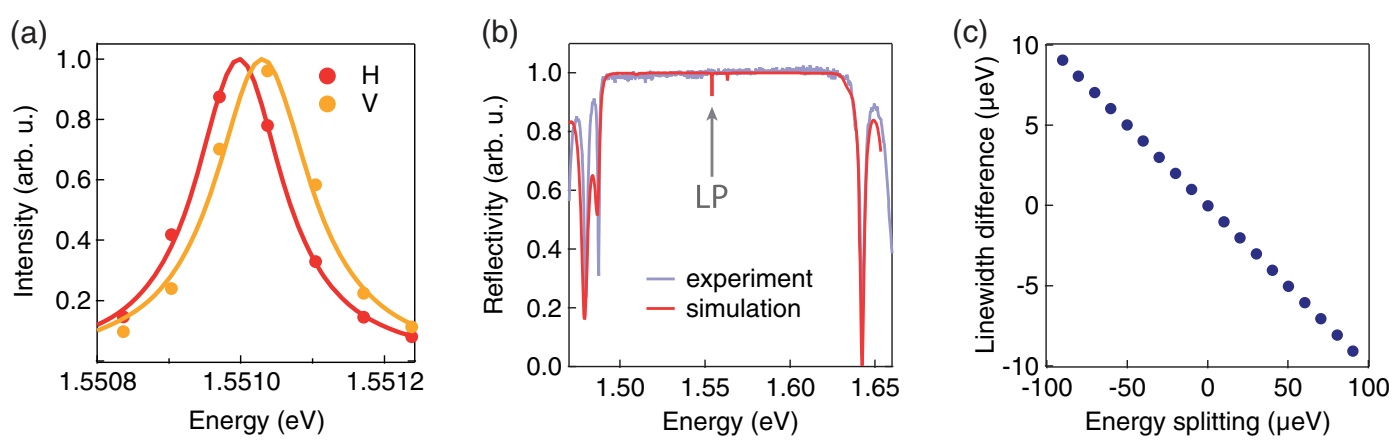

FIG. 10. (a) Energy-resolved emission of the ground-state polariton for horizontal (H) and vertical (V) polarizations, split in energy by around $30 \mu \mathrm{eV}$. (b) Transfer matrix calculations (red) and experimental reflectivity of the sample. The lower polariton mode (marked by an arrow) is offset by $10 \mathrm{meV}$ below the center of the stop band. (c) Transfer matrix calculations showing the linewidth difference versus the energy splitting. The linewidth difference is $10 \%$ of the energy splitting.

Because of the finite curvature of the cavity stop band, splitting the lower polariton into two orthogonal modes necessarily induces a difference of their linewidths as well. For the microcavity structures studied in this work, the polariton modes are located on the low-energy side of the stop band [Fig. 10(b)]. In this case, the mode possessing higher energy will exhibit a narrower linewidth since it is located closer to the stop-band center, where the DBR reflectivity is at its maximum. Correspondingly, the lower energy mode will exhibit a larger linewidth since it is located closer to the edge of the stop band, where the DBR reflectivity starts to drop. For an energy splitting of the order of $30 \mu \mathrm{eV}$, transfer matrix calculations predict a linewidth difference of approximately $3 \mu \mathrm{eV}$ [Fig. 10(c)].

This small difference in linewidth is not resolvable with our instruments. However, it should be noted that any nonzero linewidth difference, as long as it is negative relative to the energy splitting, eventually leads to the bifurcation of the circular polarization at a critical threshold [Fig. 11(a)]. Moreover, if the ratio of the linewidth difference to energy splitting is kept constant (as we have $\gamma=$ $0.1 \varepsilon$ according to our transfer matrix simulations), the condensate becomes linearly polarized at high energy splittings [Fig. 11(b)]. This result is indeed what we
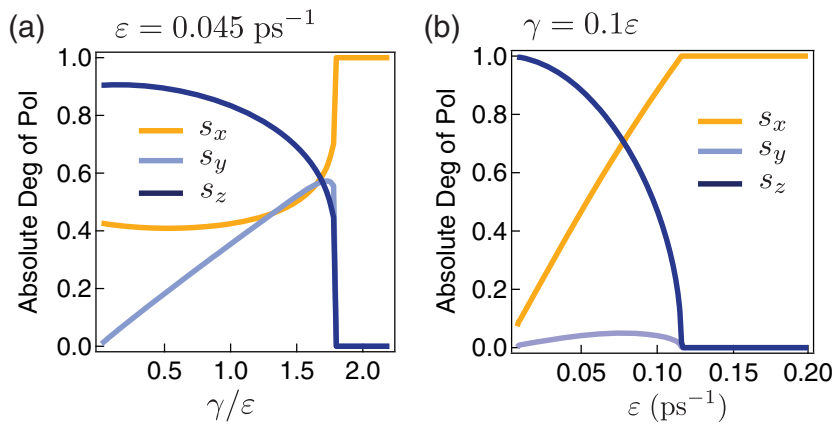

FIG. 11. (a) Absolute degree of polarization vs $\gamma$ for a fixed energy splitting of $\varepsilon=0.045 \mathrm{ps}^{-1}$. (b) Absolute degree of polarization vs energy splitting $\varepsilon$, for the case when $\gamma=0.1 \varepsilon$. observe at the edges of the membrane, where the energy splitting is as high as $100 \mu \mathrm{eV}$.

\section{APPENDIX C: VARIABLE CROSS-SPIN SATURATION}

For the case in which the same-spin and cross-spin gainsaturation nonlinearities are different, we have

$$
\begin{aligned}
\dot{\psi}_{+1}= & \frac{1}{2}\left[w-\frac{1}{2}\left(\mu\left|\psi_{+1}\right|^{2}+(2 \eta-\mu)\left|\psi_{-1}\right|^{2}\right)\right] \psi_{+1} \\
& -\frac{1}{2}(\gamma-\mathrm{i} \varepsilon) \psi_{-1}-\frac{\mathrm{i}}{2}\left[\alpha_{1}\left|\psi_{+1}\right|^{2}+\alpha_{2}\left|\psi_{-1}\right|^{2}\right] \psi_{+1}, \\
\dot{\psi}_{-1}= & \frac{1}{2}\left[w-\frac{1}{2}\left(\mu\left|\psi_{-1}\right|^{2}+(2 \eta-\mu)\left|\psi_{+1}\right|^{2}\right)\right] \psi_{-1} \\
& -\frac{1}{2}(\gamma-\mathrm{i} \varepsilon) \psi_{+1}-\frac{\mathrm{i}}{2}\left[\alpha_{1}\left|\psi_{-1}\right|^{2}+\alpha_{2}\left|\psi_{+1}\right|^{2}\right] \psi_{-1},
\end{aligned}
$$

where $w=W-\Gamma$ and we now have two saturation parameters, $\eta$ and $\mu$. The saturation is controlled by individual occupations of circular components when $\mu=2 \eta$, and the saturation is controlled by the total occupation when $\mu=\eta$. In general, $\eta \leq \mu \leq 2 \eta$. The equations above, in the matrix form, read

$$
\begin{aligned}
\frac{d \Psi}{d t}= & \frac{1}{2}\left[w-\eta S-(\mu-\eta) S_{z} \sigma_{z}\right] \Psi-\frac{1}{2}(\gamma-\mathrm{i} \varepsilon) \sigma_{x} \Psi \\
& -\frac{\mathrm{i}}{2}\left[\left(\alpha_{1}+\alpha_{2}\right) S+\left(\alpha_{1}-\alpha_{2}\right) S_{z} \sigma_{z}\right] \Psi
\end{aligned}
$$

and the equations for pseudospin components are

$$
\begin{aligned}
& \dot{S}_{x}=(w-\eta S) S_{x}-\gamma S-\alpha S_{z} S_{y}, \\
& \dot{S}_{y}=(w-\eta S) S_{y}+\varepsilon S_{z}+\alpha S_{z} S_{x},
\end{aligned}
$$




$$
\dot{S}_{z}=(w-\mu S) S_{z}-\varepsilon S_{y} .
$$

\section{Linearly polarized condensates (parity conserved)}

These particular solutions are given by $S_{y}=S_{z}=0$, $S_{x}= \pm S$, and $S=(w \mp \gamma) / \eta . X$-polarized condensates (upper sign) can exist for $W>\Gamma+\gamma . \quad Y$-polarized states (lower sign) appear for $W>\Gamma-\gamma$. Considering their stability with respect to small fluctuations, $S_{y}=y, S_{z}=z$, $S_{x}= \pm S_{0}+x$, and $S=S_{0} \mp x$, with $S_{0}=(w \mp \gamma) / \eta$, we have linearized equations

$$
\begin{aligned}
& \dot{x}=-\eta S_{0} x, \\
& \dot{y}= \pm \gamma y+\left(\varepsilon \pm \alpha S_{0}\right) z, \\
& \dot{z}=\left( \pm \gamma-(\mu-\eta) S_{0}\right) z-\varepsilon y .
\end{aligned}
$$

Taking $x, y, z \propto e^{\lambda t}$, we see that fluctuations in $x$ always decay, while Eqs. $(\mathrm{C} 4 \mathrm{~b})$ and $(\mathrm{C} 4 \mathrm{c})$ produce the equation for the Lyapunov exponent $\lambda$,

$$
(\lambda \mp \gamma)^{2}+(\mu-\eta) S_{0}(\lambda \mp \gamma)+\varepsilon(\varepsilon \pm \alpha S)=0 .
$$

The stability of the $Y$-polarized condensate is lost when one root of this equation crosses zero. This loss of stability corresponds to the critical occupation $S_{c}$ and critical pumping $W_{2}$,

$$
S_{c}=\frac{\gamma^{2}+\varepsilon^{2}}{[\alpha \varepsilon-(\mu-\eta) \gamma]}, \quad W_{2}=\Gamma-\gamma+\eta S_{c} .
$$

The stability of the $X$-polarized state depends on the interrelation between $\gamma$ and $(\mu-\eta) S_{0}$. For $\mu=\eta$, this state is always unstable. However, if $\mu>\eta$, the stable $X$ polarized state can be formed for large enough condensate occupations (far above the threshold).

\section{Elliptically polarized condensates (parity broken)}

These solutions with $S_{z} \neq 0$ are given by

$$
\begin{aligned}
& S_{x}=-\frac{1}{\alpha \varepsilon}\left[\varepsilon^{2}+(w-\eta S)(w-\mu S)\right], \\
& S_{y}=\frac{(w-\mu S)}{\varepsilon} S_{z}, \\
& S_{z}= \pm \varepsilon \sqrt{\frac{S^{2}-S_{x}^{2}}{\varepsilon^{2}+(w-\mu S)^{2}}} .
\end{aligned}
$$

Substitution into $\mathrm{Eq}(\mathrm{C} 3 \mathrm{a})$ gives

$$
\begin{aligned}
& (\mu-\eta) \varepsilon\left[\varepsilon^{2}+(w-\eta S)(w-\mu S)\right] \\
& \quad+\alpha^{2} \varepsilon(w-\mu S) S+\alpha \gamma\left[\varepsilon^{2}+(w-\mu S)^{2}\right]=0 .
\end{aligned}
$$

The positive root of this equation for $S$ should be taken. Also, it is necessary to satisfy the condition $\left|S_{x}\right| \leq S$, which gives $w \geq\left(W_{2}-\Gamma\right)=w_{2}$. This means that the weak lasing solutions appear continuously from the $Y$-polarized solution at the critical pumping $W_{2}$.

The stability of weak lasing states can also be lost. Numerical analysis shows the following typical scenario of evolution of the condensate polarization state with increasing $w$ for $\mu>\eta$. First, the $Y$ linearly polarized state is formed. Then, it transforms into a weak lasing, elliptically polarized state. The stability of the latter is also lost with increasing $w$, resulting in some irregular, quasichaotic dynamics and/or in the oscillatory motion of the pseudospin vector. Finally, for large $w$, the stable $X$-polarized state is formed.

\section{APPENDIX D: ESTIMATE FOR PARTICLE NUMBER}

The condensate particle number is experimentally measured by

$$
n=\frac{\Phi \tau}{|C|^{2}}
$$

where $\Phi$ is the photon flux, $\tau=10 \mathrm{ps}$ is the polariton lifetime, and $|C|^{2}=0.4$ is the photon Hopfield coefficient. The photon flux is measured by

$$
\Phi=\frac{\alpha R}{\eta},
$$

where $\alpha=3.5 \mathrm{e}^{-} /$count is the photoelectron sensitivity of the CCD, $\eta=0.0021$ is the total detection efficiency including the camera quantum efficiency and the total optical transmission efficiencies, and $R=1.9 \times 10^{10} \mathrm{~s}^{-1}$ is the spatially integrated count rate of the CCD. Inserting these values in Eq. (D1) gives the particle number $n \simeq 800$, at $P=1.7 P_{\text {th }}$.

\section{APPENDIX E: RESONANCE WIDTH}

We study the reduction of the opposite component of the condensate circular polarization to the gate laser [marked by $\Delta s_{-}$in Fig. 7(d)] as the "detuning" of the gate varies while the gate power remains constant. In order to change the detuning of the gate laser frequency with respect to the condensate, instead of changing the frequency of the laser, we tune the condensate frequency by changing the pump intensity. The trapped condensate "blueshifts" as the pump intensity is increased, mainly because of the repulsive interaction between the polaritons. This result contrasts with the case of untrapped condensates, where interactions of polaritons with the excitons in the reservoir are the source of blueshifts $[32,33,67]$. In the four-spot trapped geometry, we have a blueshift of $6 \mu \mathrm{eV} / \mathrm{mW}$, and as a result, we can tune the condensate energy accurately with respect to that of the 

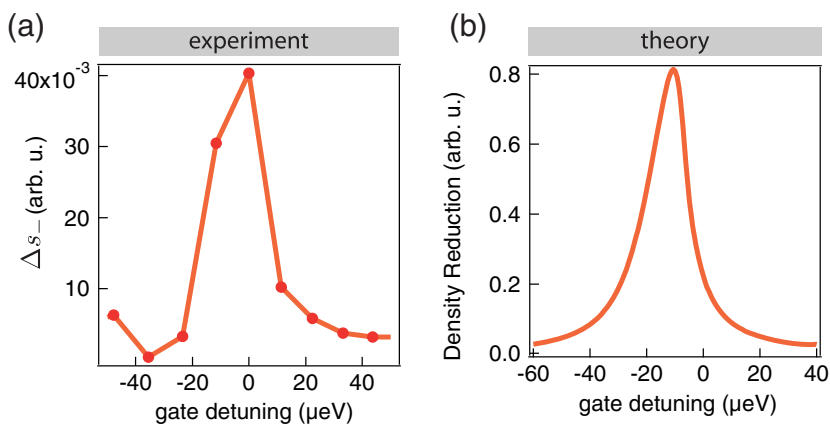

FIG. 12. (a) The reduction of the spin-down intensity vs the detuning of the gate laser frequency is shown. A sharp resonance is visible when the laser is in resonance with the condensate. (b) The numerical calculations show a similar resonance effect, with the center of the peak slightly redshifted because of the linear polarization energy splitting $\varepsilon$.

gate. Figure 12(a) shows $\Delta s_{-}$with respect to the detuning of the gate laser. We observe a sharp resonance, with a full width at half maximum (FWHM) of $11 \pm 6 \mu \mathrm{eV}$. Figure 12(b) shows the theoretical curve versus the gate detuning $\left(\hbar \omega_{g}\right)$, which resembles a Lorentzian profile with a linewidth of $17 \mu \mathrm{eV}$. Theoretically, the linewidth of the resonance is defined by the noise in Eqs. (8). The FWHM of the Lorentzian resonance peak is $D /\left(1-s_{z}^{2}\right) n$. We note that the resonance frequency $\omega_{c}$ is slightly redshifted because of the effect of the linear polarization splitting and the decay rate splitting,

$$
\omega_{c}=\frac{1}{2}\left[\left(\alpha_{1}+\alpha_{2}\right) S-\frac{\left(\gamma S_{y} S_{z}+\varepsilon S S_{x}\right)}{\left(S_{x}^{2}+S_{y}^{2}\right)}\right] .
$$

\section{APPENDIX F: KRAMERS FLIP RATE}

From Eq. (8), we can obtain the spin vector equations

$$
\begin{aligned}
& \dot{S}_{x}=-g(S) S_{x}-\gamma S-\alpha S_{z} S_{y}+F_{x}(t), \\
& \dot{S}_{y}=-g(S) S_{y}+\varepsilon S_{z}+\alpha S_{z} S_{x}+F_{y}(t), \\
& \dot{S}_{z}=-g(S) S_{z}-\varepsilon S_{y}+F_{z}(t),
\end{aligned}
$$

where the correlators of real-function noise $F_{i}(t)$ are

$$
\left\langle F_{i}(t) F_{j}\left(t^{\prime}\right)\right\rangle=2 D S \delta_{i j} \delta\left(t-t^{\prime}\right), \quad i, j=x, y, z .
$$

Here, we consider the limiting case when two parity breaking states are formed near the north and south poles of the Poincaré sphere, i.e., $\left|S_{z}\right| \gg\left|S_{x, y}\right|$. The spin components of fixed states in this limit are $S_{x 0} \simeq-\varepsilon / \alpha$, $S_{y 0} \simeq-\gamma / \alpha, \quad S_{z} \simeq \pm S_{0}$, where $S_{0}$ is the root of $S=\gamma \varepsilon / \alpha g(S)$. In what follows, we also denote $g_{0}=$ $g\left(S_{0}\right)$ and, by assumption, $g_{0} \ll \varepsilon, \gamma$. Being excited away from the fixed state, the spin exhibits fast self-induced Larmor precession and slow relaxation. The precession frequency is $\omega=\alpha S_{z}$, so that $\omega=\gamma \varepsilon / g_{0}$ near the stationary states.

The spin should be driven by noise into the equatorial plane $\left(S_{z}=0\right)$ in order to flip. This Kramers problem can be simplified if we perform averaging over fast precession of the spin vector. This treatment is valid as long as $\left|S_{z}\right| \gg\left|S_{x, y}\right|$. Consider the motion in the northern hemisphere. We assume that the number of polaritons does not change during the flip (i.e., $S$ is fixed to $S_{0}$ ), and we consider the case of large occupations, $\ln S_{0} \gg 1$. Omitting the $g$ terms from Eqs. (F1a) and (F1b), we find that for a given value of $S_{z}>0$, the averages over one cycle of the other two components are $\left\langle S_{x}\right\rangle=-\varepsilon / \alpha$ and $\left\langle S_{y}\right\rangle=-\gamma S_{0} / \alpha S_{z}$. Then, from Eq. (F1c), we obtain the equation for slow evolution of $S_{z}$,

$\frac{d S_{z}}{d t}=-g_{0} S_{z}+g_{0} \frac{S_{0}^{2}}{S_{z}}+F_{z}(t)=-\frac{d U\left(S_{z}\right)}{d S_{z}}+F_{z}(t)$,

$U\left(S_{z}\right)=g_{0}\left(\frac{1}{2} S_{z}^{2}-S_{0}^{2} \ln S_{z}\right)$

This expression for the effective potential $U\left(S_{z}\right)$ is not valid for small $S_{z}$, where it diverges logarithmically. The top of the barrier should be cut off when $S_{z}$ becomes comparable to $\left|\left\langle S_{y}\right\rangle\right|$, that is, for $S_{z} \simeq S_{0} \sqrt{g_{0} / \varepsilon}$. The value of the potential on the top of the barrier is then $U_{b} \simeq-g_{0} S_{0}^{2} \ln \left(S_{0} \sqrt{g_{0} / \varepsilon}\right)$. The bottom of the well is positioned at $U_{0}=g_{0} S_{0}^{2}\left(\frac{1}{2}-\ln S_{0}\right)$. As a result, the spin should overcome the barrier,

$$
\begin{aligned}
\Delta U=U_{b}-U_{0} & =\frac{1}{2} g_{0} S_{0}^{2}\left[\ln \left(\frac{\varepsilon}{g_{0}}\right)-1\right] \\
& \simeq \frac{1}{2} g_{0} S_{0}^{2} \ln \left(\frac{\varepsilon}{g_{0}}\right) .
\end{aligned}
$$

Using the known result for the Kramers first passage time in the one-dimensional problem [68], we obtain the spin-flip rate

$$
\begin{aligned}
R_{K}=\frac{R_{0}}{2 \pi} \exp \left\{-\frac{\Delta U}{D S_{0}}\right\} & =\frac{R_{0}}{2 \pi} \exp \left\{-\frac{g_{0} S_{0}}{2 D} \ln \left(\frac{\varepsilon}{g_{0}}\right)\right\}, \\
S_{0} & =\frac{\gamma \varepsilon}{\alpha g_{0}} .
\end{aligned}
$$

This expression assumes $g_{0} S_{0} / D \gg 1$ and $\varepsilon \gg g_{0}$. The preexponent $R_{0}$ cannot be written exactly by this method since we do not know the shape of the effective potential near the top of the barrier. It can be estimated as $R_{0} \simeq \sqrt{\varepsilon g_{0}}$. 


\section{APPENDIX G: SIMULATIONS AND NUMERICAL PARAMETERS}

The parameters used for all the simulations are as follows: 0D simulations: $\eta=0.01 \mathrm{ps}^{-1}, \Gamma=0.1 \mathrm{ps}^{-1}$, $\hbar \varepsilon=30 \mu \mathrm{eV}, \gamma=0.1 \varepsilon, \hbar \alpha_{1}=10 \mu \mathrm{eV}, \alpha_{2}=-0.5 \alpha_{1} ; 2 \mathrm{D}$ simulations: $\quad \hbar \alpha_{1}=3 \mu \mathrm{eV} \mu \mathrm{m}^{2}, \quad \alpha_{2}=-0.5 \alpha_{1}, \quad \hbar g_{r}=$ $46 \mu \mathrm{eV} \mu \mathrm{m}^{2}, \quad g_{P}=g_{R} / 4, \quad \Lambda=0.1, \quad m^{*}=5.1 \times 10^{-5} m_{e}$, $\gamma_{R}=10 \mathrm{ps}^{-1} \mu \mathrm{m}^{2}, \hbar \varepsilon=7 \mu \mathrm{eV}, \gamma=0.2 \varepsilon$.

[1] H. Deng, G. Weihs, C. Santori, J. Bloch, and Y. Yamamoto, Condensation of semiconductor microcavity exciton polaritons, Science 298, 199 (2002).

[2] J. Kasprzak, M. Richard, S. Kundermann, A. Baas, P. Jeambrun, J. M. J. Keeling, F. M. Marchetti, M. H. Szymanska, R. André, J. L. Staehli, V. Savona, P. B. Littlewood, B. Deveaud, and L. S. Dang, Bose-Einstein condensation of exciton polaritons, Nature (London) 443, 409 (2006).

[3] R. Balili, V. Hartwell, D. Snoke, L. Pfeiffer, and K. West, Bose-Einstein condensation of microcavity polaritons in a trap, Science 316, 1007 (2007).

[4] J. Baumberg, A. Kavokin, S. Christopoulos, A. Grundy, R. Butté, G. Christmann, D. Solnyshkov, G. Malpuech, G. Baldassarri Höger von Högersthal, E. Feltin, J. F Carlin, and N. Grandjean, Spontaneous Polarization Buildup in a Room-Temperature Polariton Laser, Phys. Rev. Lett. 101, 136409 (2008).

[5] H. Ohadi, E. Kammann, T. C. H. Liew, K. G. Lagoudakis, A. V. Kavokin, and P. G. Lagoudakis, Spontaneous Symmetry Breaking in a Polariton and Photon Laser, Phys. Rev. Lett. 109, 016404 (2012).

[6] B. Nelsen, G. Liu, M. Steger, D. W. Snoke, R. Balili, K. West, and L. Pfeiffer, Dissipationless Flow and Sharp Threshold of a Polariton Condensate with Long Lifetime, Phys. Rev. X 3, 041015 (2013).

[7] C. Sturm, D. Tanese, H. S. Nguyen, H. Flayac, E. Galopin, A. Lemaître, I. Sagnes, D. Solnyshkov, A. Amo, G. Malpuech, and J. Bloch, All-optical phase modulation in a cavity-polariton Mach-zehnder interferometer, Nat. Commun. 5, 3278 (2014).

[8] A. Dreismann, P. Cristofolini, R. Balili, G. Christmann, F. Pinsker, N. G. Berloff, Z. Hatzopoulos, P. G. Savvidis, and J. J. Baumberg, Coupled counterrotating polariton condensates in optically defined annular potentials, Proc. Natl. Acad. Sci. U.S.A. 111, 8770 (2014).

[9] A. Amo, T. C.H. Liew, C. Adrados, R. Houdre, E. Giacobino, A. V. Kavokin, and A. Bramati, Excitonpolariton spin switches, Nat. Photonics 4, 361 (2010).

[10] D. Ballarini, M. De Giorgi, E. Cancellieri, R. Houdré, E. Giacobino, R. Cingolani, A. Bramati, G. Gigli, and D. Sanvitto, All-optical polariton transistor, Nat. Commun. 4 , 1778 (2013).

[11] R. Cerna, Y. Léger, T. K. Paraïso, M. Wouters, F. Morier-Genoud, M. T. Portella-Oberli, and B. Deveaud, Ultrafast tristable spin memory of a coherent polariton gas, Nat. Commun. 4, 2008 (2013).
[12] H. S. Nguyen, D. Vishnevsky, C. Sturm, D. Tanese, D. Solnyshkov, E. Galopin, A. Lemaître, I. Sagnes, A. Amo, G. Malpuech, and J. Bloch, Realization of a Double-Barrier Resonant Tunneling Diode for Cavity Polaritons, Phys. Rev. Lett. 110, 236601 (2013).

[13] P. Bhattacharya, B. Xiao, A. Das, S. Bhowmick, and J. Heo, Solid State Electrically Injected Exciton-Polariton Laser, Phys. Rev. Lett. 110, 206403 (2013).

[14] C. Schneider, A. Rahimi-Iman, N. Young Kim, J. Fischer, I. G. Savenko, M. Amthor, M. Lermer, A. Wolf, L. Worschech, V. D. Kulakovskii, I. A. Shelykh, M. Kamp, S. Reitzenstein, A. Forchel, Y. Yamamoto, and S. Höfling, An electrically pumped polariton laser, Nature (London) 497, 348 (2013).

[15] I. Buluta and F. Nori, Quantum Simulators, Science 326, 108 (2009).

[16] I. L. Aleiner, B. L. Altshuler, and Y. G. Rubo, Radiative coupling and weak lasing of exciton-polariton condensates, Phys. Rev. B 85, 121301 (2012).

[17] L. Zhang, W. Xie, J. Wang, A. Poddubny, J. Lu, Y. Wang, J. Gu, W. Liu, D. Xu, X. Shen, Y. G. Rubo, B. L. Altshuler, A. V. Kavokin, and Z. Chen, Weak lasing in onedimensional polariton superlattices, Proc. Natl. Acad. Sci. U.S.A. 112, E1516 (2015).

[18] P. G. Lagoudakis, P. G. Savvidis, J. J. Baumberg, D. M. Whittaker, P. R. Eastham, M. S. Skolnick, and J. S. Roberts, Stimulated spin dynamics of polaritons in semiconductor microcavities, Phys. Rev. B 65, 161310 (2002).

[19] M. D. Martín, G. Aichmayr, L. Viña, and R. André, Polarization Control of the Nonlinear Emission of Semiconductor Microcavities, Phys. Rev. Lett. 89, 077402 (2002).

[20] A. Kavokin, P. G. Lagoudakis, G. Malpuech, and J. J. Baumberg, Polarization rotation in parametric scattering of polaritons in semiconductor microcavities, Phys. Rev. B 67, 195321 (2003).

[21] K. V. Kavokin, I. A. Shelykh, A. V. Kavokin, G. Malpuech, and P. Bigenwald, Quantum Theory of Spin Dynamics of Exciton-Polaritons in Microcavities, Phys. Rev. Lett. 92, 017401 (2004).

[22] P. Renucci, T. Amand, X. Marie, P. Senellart, J. Bloch, B. Sermage, and K. V. Kavokin, Microcavity polariton spin quantum beats without a magnetic field: A manifestation of Coulomb exchange in dense and polarized polariton systems, Phys. Rev. B 72, 075317 (2005).

[23] D. N. Krizhanovskii, D. Sanvitto, I. A. Shelykh, M. M. Glazov, G. Malpuech, D. D. Solnyshkov, A. Kavokin, S. Ceccarelli, M. S. Skolnick, and J. S. Roberts, Rotation of the plane of polarization of light in a semiconductor microcavity, Phys. Rev. B 73, 073303 (2006).

[24] N. A. Gippius, I. A. Shelykh, D. D. Solnyshkov, S. S. Gavrilov, Y. G. Rubo, A. V. Kavokin, S. G. Tikhodeev, and G. Malpuech, Polarization Multistability of Cavity Polaritons, Phys. Rev. Lett. 98, 236401 (2007).

[25] C. Leyder, M. Romanelli, J. Ph Karr, E. Giacobino, T. C. H. Liew, M. M. Glazov, A. V. Kavokin, G. Malpuech, and A. Bramati, Observation of the optical spin Hall effect, Nat. Phys. 3, 628 (2007).

[26] T. K. Paraïso, M. Wouters, Y. Léger, F. Morier-Genoud, and B. Deveaud-Plédran, Multistability of a coherent spin 
ensemble in a semiconductor microcavity, Nat. Mater. 9, 655 (2010).

[27] T. Gao, P. S. Eldridge, T. C. H. Liew, S. I. Tsintzos, G. Stavrinidis, G. Deligeorgis, Z. Hatzopoulos, and P. G. Savvidis, Polariton condensate transistor switch, Phys. Rev. B 85, 235102 (2012).

[28] E. Kammann, T. C. H. Liew, H. Ohadi, P. Cilibrizzi, P. Tsotsis, Z. Hatzopoulos, P. G. Savvidis, A. V. Kavokin, and P. G. Lagoudakis, Nonlinear Optical Spin Hall Effect and Long-Range Spin Transport in Polariton Lasers, Phys. Rev. Lett. 109, 036404 (2012).

[29] N. Takemura, S. Trebaol, M. Wouters, M. T. Portella-Oberli, and B. Deveaud, Polaritonic Feshbach resonance, Nat. Phys. 10, 500 (2014).

[30] A. V. Kavokin, J. Baumberg, G. Malpuech, and F. P. Laussy, Microcavities (Oxford University Press, Oxford, 2007).

[31] P. G. Savvidis, J. J. Baumberg, R. M. Stevenson, M. S. Skolnick, D. M. Whittaker, and J. S. Roberts, AngleResonant Stimulated Polariton Amplifier, Phys. Rev. Lett. 84, 1547 (2000).

[32] E. Wertz, L. Ferrier, D. D. Solnyshkov, R. Johne, D. Sanvitto, A. Lemaître, I. Sagnes, R. Grousson, A. V. Kavokin, P. Senellart, G. Malpuech, and J. Bloch, Spontaneous formation and optical manipulation of extended polariton condensates, Nat. Phys. 6, 860 (2010).

[33] A. Askitopoulos, H. Ohadi, A. V. Kavokin, Z. Hatzopoulos, P. G. Savvidis, and P. G. Lagoudakis, Polariton condensation in an optically induced two-dimensional potential, Phys. Rev. B 88, 041308 (2013).

[34] I. Bloch, Ultracold quantum gases in optical lattices, Nat. Phys. 1, 23 (2005).

[35] M. Wouters and I. Carusotto, Excitations in a Nonequilibrium Bose-Einstein Condensate of Exciton Polaritons, Phys. Rev. Lett. 99, 140402 (2007).

[36] G. Tosi, G. Christmann, N. G. Berloff, P. Tsotsis, T. Gao, Z. Hatzopoulos, P. G. Savvidis, and J. J. Baumberg, Sculpting oscillators with light within a nonlinear quantum fluid, Nat. Phys. 8, 190 (2012).

[37] I. A. Shelykh, Y. G. Rubo, G. Malpuech, D. D. Solnyshkov, and A. Kavokin, Polarization and Propagation of Polariton Condensates, Phys. Rev. Lett. 97, 066402 (2006).

[38] I. Shelykh, K. V. Kavokin, A. V. Kavokin, G. Malpuech, P. Bigenwald, H. Deng, G. Weihs, and Y. Yamamoto, Semiconductor microcavity as a spin-dependent optoelectronic device, Phys. Rev. B 70, 035320 (2004).

[39] G. Roumpos, C.-W. Lai, T. C. H. Liew, Y. G. Rubo, A. V. Kavokin, and Y. Yamamoto, Signature of the microcavity exciton-polariton relaxation mechanism in the polarization of emitted light, Phys. Rev. B 79, 195310 (2009).

[40] G. Li, T. C. H. Liew, O. A. Egorov, and E. A. Ostrovskaya, Incoherent Excitation and Switching of Spin States in Exciton-Polariton Condensates, arXiv:1501.05355.

[41] Y. G. Rubo, A. V. Kavokin, and I. A. Shelykh, Suppression of superfluidity of exciton-polaritons by magnetic field, Phys. Lett. A 358, 227 (2006).

[42] M. Vladimirova, S. Cronenberger, D. Scalbert, K. V. Kavokin, A. Miard, A. Lemaître, J. Bloch, D. Solnyshkov, G. Malpuech, and A. V. Kavokin, Polariton-polariton interaction constants in microcavities, Phys. Rev. B 82, 075301 (2010).

[43] N. Takemura, S. Trebaol, M. Wouters, M. T. Portella-Oberli, and B. Deveaud, Heterodyne spectroscopy of polariton spinor interactions, Phys. Rev. B 90, 195307 (2014).

[44] J. Kasprzak, D. D. Solnyshkov, R. André, Le Si Dang, and G. Malpuech, Formation of an Exciton Polariton Condensate: Thermodynamic Versus Kinetic Regimes, Phys. Rev. Lett. 101, 146404 (2008).

[45] R. Balili, B. Nelsen, D. W. Snoke, R. H. Reid, L. Pfeiffer, and K. West, Huge splitting of polariton states in microcavities under stress, Phys. Rev. B 81, 125311 (2010).

[46] Ł. Klopotowski, M. D. Martín, A. Amo, L. Viña, I. A. Shelykh, M. M. Glazov, G. Malpuech, A. V. Kavokin, and R. André, Optical anisotropy and pinning of the linear polarization of light in semiconductor microcavities, Solid State Commun. 139, 511 (2006).

[47] A. Kavokin, G. Malpuech, and M. Glazov, Optical Spin Hall Effect, Phys. Rev. Lett. 95, 136601 (2005).

[48] P. Cristofolini, A. Dreismann, G. Christmann, G. Franchetti, N. G. Berloff, P. Tsotsis, Z. Hatzopoulos, P. G. Savvidis, and J. J. Baumberg, Optical Superfluid Phase Transitions and Trapping of Polariton Condensates, Phys. Rev. Lett. 110, 186403 (2013).

[49] A. Askitopoulos, T. C. H. Liew, H. Ohadi, Z. Hatzopoulos, P. G. Savvidis, and P. G. Lagoudakis, A Robust Platform for Engineering Pure-Quantum-State Transitions in Polariton Condensates, arXiv:1411.4579.

[50] J. Keeling and N. G. Berloff, Spontaneous Rotating Vortex Lattices in a Pumped Decaying Condensate, Phys. Rev. Lett. 100, 250401 (2008).

[51] K. Rayanov, B. L. Altshuler, Y. G. Rubo, and S. Flach, Frequency Combs with Weakly Lasing Exciton-Polariton Condensates, Phys. Rev. Lett. 114, 193901 (2015).

[52] M. Wouters, T. C. H. Liew, and V. Savona, Energy relaxation in one-dimensional polariton condensates, Phys. Rev. B 82, 245315 (2010).

[53] L. E. Sadler, J. M. Higbie, S. R. Leslie, M. Vengalattore, and D. M. Stamper-Kurn, Spontaneous symmetry breaking in a quenched ferromagnetic spinor Bose-Einstein condensate, Nature (London) 443, 312 (2006).

[54] D. M. Stamper-Kurn and M. Ueda, Spinor Bose gases: Symmetries, magnetism, and quantum dynamics, Rev. Mod. Phys. 85, 1191 (2013).

[55] N. V. Alexeeva, I. V. Barashenkov, K. Rayanov, and S. Flach, Actively coupled optical waveguides, Phys. Rev. A 89, 013848 (2014).

[56] K. Nozaki, T. Tanabe, A. Shinya, S. Matsuo, T. Sato, H. Taniyama, and M. Notomi, Sub-Femtojoule all-optical switching using a photonic-crystal nanocavity, Nat. Photonics 4, 477 (2010).

[57] M. Wouters, T. K. Paraïso, Y. Léger, R. Cerna, F. MorierGenoud, M. T. Portella-Oberli, and B. Deveaud-Plédran, Influence of a nonradiative reservoir on polariton spin multistability, Phys. Rev. B 87, 045303 (2013).

[58] M. T. Hill, H. J. S. Dorren, T. de Vries, X. J. M. Leijtens, J. H. den Besten, B. Smalbrugge, Y.-S. Oei, H. Binsma, G.-D. Khoe, and M. K. Smit, A fast low-power 
optical memory based on coupled micro-ring lasers, Nature 432, 206 (2004).

[59] L. Liu, R. Kumar, K. Huybrechts, T. Spuesens, G. Roelkens, E.-J. Geluk, T. de Vries, P. Regreny, D. Van Thourhout, R. Baets, and G. Morthier, An ultra-small, low-power, alloptical flip-flop memory on a silicon chip, Nat. Photonics 4, 182 (2010).

[60] D. Read, T. C. H. Liew, Y. G. Rubo, and A. V. Kavokin, Stochastic polarization formation in exciton-polariton Bose-Einstein condensates, Phys. Rev. B 80, 195309 (2009).

[61] K. G. Lagoudakis, B. Pietka, M. Wouters, R. André, and B. Deveaud-Plédran, Coherent Oscillations in an ExcitonPolariton Josephson Junction, Phys. Rev. Lett. 105, 120403 (2010).

[62] M. Jamet, W. Wernsdorfer, C. Thirion, D. Mailly, V. Dupuis, P. Mélinon, and A. Pérez, Magnetic Anisotropy of a Single Cobalt Nanocluster, Phys. Rev. Lett. 86, 4676 (2001).
[63] P. N. Hai, S. Ohya, and M. Tanaka, Long spin-relaxation time in a single metal nanoparticle, Nat. Nanotechnol. 5, 593 (2010).

[64] P. Tsotsis, P. S. Eldridge, T. Gao, S. I. Tsintzos, Z. Hatzopoulos, and P. G. Savvidis, Lasing threshold doubling at the crossover from strong to weak coupling regime in GaAs microcavity, New J. Phys. 14, 023060 (2012).

[65] I. L. Aleiner and E. L. Ivchenko, Anisotropic exchange splitting in type-II GaAs/AlAs superlattices, JETP Lett. 55, 692 (1992).

[66] E. L. Ivchenko, A. Yu. Kaminski, and U. Rössler, Heavylight hole mixing at zinc-blende (001) interfaces under normal incidence, Phys. Rev. B 54, 5852 (1996).

[67] M. Wouters, I. Carusotto, and C. Ciuti, Spatial and spectral shape of inhomogeneous nonequilibrium exciton-polariton condensates, Phys. Rev. B 77, 115340 (2008).

[68] R. Zwanzig, Nonequilibrium Statistical Mechanics (Oxford University Press, Oxford, New York, 2001). 